Revista de la Escuela de Ciencias de la Educación, año 12, nRo. 11, vol. 2, Julio a diciembre de 2016. Páginas 155-176. ISSN 1851-6297. ISSN 2362-3349 (EN LINNEA). LA PRÁCTICA DOCENTE MÁS ALLÁ DE LA DIDÁCTICA. DANIELA REYES-GASPERINI - Ricardo CANTORA

\title{
EMPODERAMIENTO DOCENTE: LA PRÁCTICA DOCENTE MÁS ALLÁ DE LA DIDÁCTICA... ¿QUÉ PAPEL JUEGA EL SABER EN UNA TRANSFORMACIÓN EDUCATIVA?
}

\author{
Daniela Reyes-Gasperini* \\ Centro de Investigación y de Estudios Avanzados, México. \\ dreyes@cinvestav.mx \\ Ricardo Cantoral** \\ Centro de Investigación y de Estudios Avanzados, México. \\ rcantor@cinvestav.mx
}

Recibido: 15/03/2016 Aceptado: 28/05/2016

\section{Resumen}

La Matemática Educativa, de origen latinoamericano y alcance mundial, concebida como disciplina científica que estudia fenómenos didácticos ligados al saber matemático, se encuentra en búsqueda continua de trayectos oportunos para la mejora en los procesos de enseñanza y de aprendizaje de las matemáticas. En este artículo compartimos uno de los más recientes estudios referente al desarrollo profesional docente desde la Teoría Socioepistemológi-

* Profesora de Matemática por el Instituto Superior del Profesorado "Dr. Joaquín V. González", Ciudad Autónoma de Buenos Aires, Argentina. Maestra y Doctora en Ciencias especialidad en Matemática Educativa por el Centro de Investigación y de Estudios Avanzados del IPN (Cinvestav-IPN), Ciudad de México, México. Becaria Doctoral del Consejo Nacional de Ciencia y Tecnología (CONACYT). Miembro del Comité Latinoamericano de Matemática Educativa y de la Sociedad Argentina de Educación Matemática.

* Investigador titular 3D del Cinvestav IPN. Doctor en la especialidad de Matemática Educativa por el Cinvestav. Realizó un posdoctorado en la Université Paris VII. Investigador nacional del Sistema Nacional de Investigadores en su máximo nivel y miembro de la Academia Mexicana de Ciencias. Obtuvo la distinción de Profesor de Mérito del Ministerio de Educación Superior, La Habana y fue acreedor a la Medalla al Mérito 45 aniversario de la Universidad de Camagüey, CUBA. Fundador y presidente del Comité Latinoamericano de Matemática Educativa - CLAME. Director de la Revista Latinoamericana de Investigación en Matemática Educativa - Relime. 
Revista de la Escuela de Ciencias de la Educación, año 12, nRo. 11, vol. 2, Julio a diciembre de 2016. Páginas 155-176. ISSN 1851-6297. ISSN 2362-3349 (EN LíNEA). LA PRÁCTICA DOCENTE MÁS ALLÁ DE LA DIDÁCTICA. DANIELA REYES-GASPERINI - RICARDO CANTORA

ca: el proceso de empoderamiento docente. Los lectores apreciarán cómo un fenómeno típicamente social, entrelazado con una mirada socioepistemológica de las matemáticas que pone en el centro a la problematización de la matemática escolar, promueve un cambio en la relación con el conocimiento matemático por parte del docente a fin de impulsar una transformación educativa.

\title{
Palabras clave:
}

Empoderamiento - Socioepistemología - Problematización de la Matemática Escolar.

\begin{abstract}
Mathematics teaching and learning are researched by Latin American Mathematics Education with global reach. This is a scientific discipline which studies didactic phenomena linked to mathematical knowledge. New and optimum learning and teaching paths are continuously investigated to better teach and learn mathematics. In this article we share one of the most recent studies on teachers professional development from the Socioepistemologic Theory: the teachers empowerment process. Readers will appreciate how a typically social phenomenon, interwoven with a socioepistemological gaze of mathematics, which puts in the center the problematization of school mathematics, promotes a change in the relation between mathematical knowledge by the teacher in order to propel an education transformation.
\end{abstract}

\section{Keywords:}

Empowerment-Socioepistemology-Problematization of School Mathematics.

\section{Introducción}

La investigación científica en Matemática Educativa se ocupa de brindar una respuesta ante situaciones específicas de la sociedad ligadas a las problemáticas propias de la dialéctica enseñanza-aprendizaje de las matemáticas.

Mientras que la pregunta best-seller de los años 90's fue sin duda el cómo enseñar mediante diversas estrategias didácticas que pudieran hacer más accesible la comprensión de ciertos conocimientos matemáticos a los estudiantes de diferentes niveles educativos, la Teoría Socioepistemológica se planteó nuevos interrogantes: ¿qué es lo que estamos enseñando?, ¿qué es aquello que nuestros estudiantes están aprendiendo? Es decir, se propuso desde sus comienzos estudiar y discutir sobre la naturaleza del saber matemático y desde allí, reflexionar sobre el saber matemático escolar: el conocimiento puesto en uso en escenarios escolares.

Ahora bien, en cuanto a la práctica docente ¿qué se estudia en esta línea 
Revista de la Escuela de Ciencias de la Educación, año 12, nRo. 11, vol. 2, Julio a diciembre de 2016. Páginas 155-176. ISSN 1851-6297. ISSN 2362-3349 (EN LINEA). LA PRÁCTICA DOCENTE MÁS ALLÁ DE LA DIDÁCTICA. DANIELA REYES-GASPERINI - Ricardo CANTORA

de investigación? He aquí la repetición de la historia: mientras que las corrientes clásicas analizan, con o sin el profesor, las tareas que los docentes usan en clase, las interacciones didácticas que tienen los estudiantes y sus profesores, la competencia puesta en juego para resolver problemas matemáticos, el conocimiento de los profesores sobre cómo los estudiantes piensan, conocen o aprenden un contenido matemático específico, entre muchas otras, la Socioepistemología se preguntó ¿cuál y cómo es la relación con el conocimiento matemático por parte del profesor de Matemáticas en una relación didáctica? Una vez más, como era de esperarse, el saber matemático adquirió mayor protagonismo en nuestro camino.

La Matemática Educativa se propone, desde sus inicios, un objetivo mayor e impostergable: alcanzar una democratización del aprendizaje de las matemáticas, esto es "que ningún ciudadano se quede atrás". Uno de los medios para lograrlo consiste en aceptar un cambio de centración que va de la mirada platónica, focalizada en objetos abstractos ajenos a la realidad, a una visión socioepistemológica que asume a las prácticas sociales como la base misma de la construcción de significado en matemáticas (Cantoral, 2013a). Esto precisó de numerosas restructuraciones teóricas y prácticas al nivel del sistema educativo, una de ellas que consideramos fundamental y de la que tratamos intensamente en el presente escrito es el asunto del desarrollo profesional docente en el campo de las matemáticas. Nuestro eje de estudio considera una articulación indispensable entre tres elementos teóricos: la funcionalidad del saber matemático sobre la proporcionalidad (noción transversal en el sistema educativo y de alto valor práctico en la vida cotidiana), el constructo teórico de empoderamiento docente y la noción misma de espacio áulico que plantea la Socioepistemología (el aula extendida). Con base en dicha articulación, tejimos un entramado conceptual a fin de mostrar que el empoderamiento docente desde una visión socioepistemológica es un proceso que estudia a la profesionalización, problematizando la matemática escolar (anteriormente, denominada problematización del saber matemático). Este, es un cambio posible; pues lo mostró la propia experiencia de trabajar conjuntamente con docentes de educación secundaria (12 a 14 años) y nivel bachillerato (15 a 18 años) en el marco de un programa institucional mexicano. Nuestra misión entonces, como investigadores en activo, fue la de explicar teóricamente aquello que la empiria nos mostraba y que posteriormente reprodujimos en diversas regiones de México con más de 500 docentes en Oaxaca, Hidalgo, Puebla, Querétaro y Zacatecas.

Los lectores podrán encontrar en estas páginas elementos que facilitaron una reflexión profunda sobre el fenómeno de empoderamiento, enfatizando los caminos futuros. Se podrán encontrar ejemplos en detalle en (Reyes-Gasperini, 2011; Reyes-Gasperini y Cantoral, 2014; Reyes-Gasperini, Cantoral y Montiel, 2013), donde se explicita cómo el empoderamiento, a causa de un 
Revista de la Escuela de Ciencias de la Educación, año 12, nRo. 11, vol. 2, Julio a diciembre de 2016. Páginas 155-176. ISSN 1851-6297. ISSN 2362-3349 (EN LINEA). LA PRÁCTICA DOCENTE MÁS ALLÁ DE LA DIDÁCTICA. DANIELA REYES-GASPERINI - RICARDO CANTORA

cambio de relación con el conocimiento matemático por parte del docente, le brinda la libertad de privilegiar la actividad situada del que aprende, su contexto de significación; le permite además el reconocer las distintas formas de argumentación que favorecen diversas racionalidades contextualizadas, y en ese sentido, propiciar la naturaleza funcional del saber, dotarlo de valor de uso. En síntesis, esto favorece un proceso de resignificación progresiva del que aprende inmerso en marcos referenciales diversos.

Invitamos a los lectores de este artículo a transitar por el camino recorrido por los autores, relativo al estudio de un fenómeno enmarcado en la línea del desarrollo profesional docente: el empoderamiento. ¿Por qué empoderar al docente? ¿En qué sentido se habla de empoderarlo? ¿Cuáles son los vínculos entre empoderamiento y saber matemático? ¿Cómo sabremos que está inmerso en un proceso de empoderamiento? Estas y otras preguntas son abordadas.

\section{¿Por qué dar vuelta la mirada hacia el saber matemático?}

Para la investigación es válido preguntarse cómo hacer más accesible un conocimiento matemático, ya sea poniendo el foco en los docentes en cuanto a las estrategias didácticas utilizadas para su enseñanza, o centrándonos en los estudiantes y el estudio de sus respuestas. Por tanto, la pregunta parece natural: ¿por qué esta vuelta de mirada y no continuar con la centración en las interacciones del docente con el estudiante?

Veamos esto mediante un ejemplo: si habláramos de la noción de función como un concepto difícil de asimilar, comprender y comunicar, también deberíamos reconocer las características específicas que tienen los diversos tipos de funciones. En particular en las funciones polinómicas (Alanís, 1996), las funciones periódicas (Buendía, 2004; Buendía y Cordero, 2005), las funciones trigonométricas (Montiel, 2011; Montiel, 2013), las funciones logarítmicas (Ferrari, 2010; Ferrari y Farfán, 2008), las funciones exponenciales (Lezama, 2003), es decir, lo que pareciera ser un "adjetivo" de las funciones, esconde un complejo conglomerado de niveles prácticos, funcionales, históricos y sociales que el sistema didáctico desconoce y aparece pura y exclusivamente expresado en la unidad temática: Funciones con distintos mecanismos de apropiación. Algo similar ocurre con las gráficas, las que en los libros de texto se muestran como ilustraciones acompañantes de la función, cuando en realidad son espacios donde se puede construir conocimiento (Cordero, Cen Che y Suárez, 2010; Arrieta, 2003; Cantoral y Montiel, 2003a, 2003b; Farfán, 2013). Del mismo modo, podríamos observar esto en el caso de la proporcionalidad (Cantoral y Reyes-Gasperini, 2012; Reyes-Gasperini, 2011; Reyes-Gasperini y Cantoral, 2014; Reyes-Gasperini, Cantoral y Montiel, 2014), siendo un tema de gran relevancia en la matemática escolar (Hart, 1988; Godino y Batanero, 2002; Oliveira, 2009) que es reducido, la mayoría de las 
Revista de la Escuela de Ciencias de la Educación, año 12, nRo. 11, vol. 2, Julio a diciembre de 2016. PÁginas 155-176. ISSN 1851-6297. ISSN 2362-3349 (EN LINEA). LA PRÁCTICA DOCENTE MÁS ALLÁ DE LA DIDÁCTICA. DANIELA REYES-GASPERINI - RICARDO CANTORA

veces, a la buena aplicación de la regla de tres simple, el valor unitario y la igualdad entre razones. Podríamos, también, focalizar nuestra atención en el Cálculo y verificar que la enseñanza se basa en la correcta aplicación de la regla de los cuatro pasos para obtener la derivada, lo cual cuestionamos, pero que en realidad, la derivada esconde un pensamiento y lenguaje variacional (Cantoral, 1990, 2013b; Caballero, 2012) que ha sido disminuido en el discurso didáctico que los manuales escolares transmiten.

Estos y otros ejemplos son los que nos hacen dar vuelta la mirada al saber matemático, pero insistimos: ¿por qué? Si partimos de la idea que señala que los problemas del aprendizaje en matemáticas residen en las acciones de los actores del sistema educativo, en particular los docentes, inevitablemente enfocaríamos nuestros análisis hacia cuestiones propiamente didáctico - pedagógicas a fin de contribuir en la mejora de las clases brindando mejores estrategias didácticas y hacer, de este modo, más accesible un saber matemático. Sin embargo, a contracorriente de lo que suele afirmarse, nuestra estrategia fue un tanto distinta; despersonificar dicha problemática y como hemos dicho, "dar vuelta la mirada" hacia aquello que hasta hace tres décadas no se cuestionaba: poner en discusión al propio objeto del aprendizaje, los contenidos y sus ideologías, digámoslo así, estudiar los efectos del discurso Matemático Escolar ( $d M E)$ sobre los aprendizajes. Partimos entonces de la consideración de que éste es el causante de la mayor resistencia para el aprendizaje de las matemáticas. Si logramos el cuestionamiento del saber matemático podremos entonces, como consecuencia, discutir con los docentes la matemática escolar expresada, por ejemplo, en propuestas didácticas que nacen desde la investigación, o bien, entender y atender las reformas educativas que sufren los sistemas educativos continuamente.

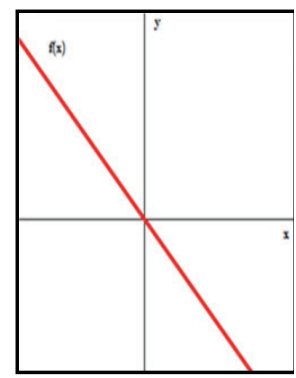

Figura 1: Gráfica de una función de proporcionalidad.

Sólo como ejemplo: es habitual escuchar que para argumentar cuándo una función es de proporcionalidad directa o inversa se diga "es directa cuando uno aumenta y el otro aumenta", y "es inversa cuando uno aumenta y el 
Revista de la Escuela de Ciencias de la Educación, año 12, nRo. 11, vol. 2, Julio a diciembre de 2016. Páginas 155-176. ISSN 1851-6297. ISSN 2362-3349 (EN LINEA). LA PRÁCTICA DOCENTE MÁS ALLÁ DE LA DIDÁCTICA. DANIELA REYES-GASPERINI - RICARDO CANTORA

otro disminuye". Éste es un estribillo en la matemática escolar que "ayuda" a los estudiantes a diferenciar una situación de otra. Ahora bien, dada esta "ayuda nemotécnica", veamos la siguiente representación gráfica de una función (ver Figura 1): ¿Es de proporcionalidad directa o inversa? Más adelante veremos cuál es la respuesta matemáticamente correcta, mientras tanto, dejamos a los lectores pensando la respuesta (Reyes-Gasperini, Cantoral y Montiel, 2014).

Los docentes, en particular hacemos referencia a los mexicanos que hoy día dan clases de Matemáticas, fueron formados, ya sea en su formación inicial o en alguna formación afín que los llevó a la profesión docente, bajo una concepción de la Matemática basada en objetos abstractos y aislados de la realidad, considerados objetos anteriores a la praxis social y, en consecuencia, externos al individuo, siendo el profesor quien debe comunicar "verdades preexistentes" a sus alumnos, en donde la construcción social del conocimiento matemático queda sorteada (Cantoral, 2003). Entonces, ¿cómo podrían los docentes cuestionarse el conocimiento matemático escolar si en la mayoría de sus acercamientos a la matemática fue presentada como un cúmulo de conceptos que están "dados" y que su misión principal es que los estudiantes los "aprendan", o quizás, "aprehendan"?

Aunado a las propuestas que se realizan sobre el desarrollo profesional del profesorado (Ponte, 2012), el proceso que se vive del análisis de la práctica al diseño de tareas matemáticas en la formación docente (Llinares, 2012), nosotros ponemos la lupa en el propio saber matemático y proponemos estudiar el desarrollo profesional docente a partir de la problematización de la matemática escolar, de suerte que esta acción les permita a los docentes hacerse dueños de su propia práctica a través del cambio de relación al conocimiento matemático, es decir, que transiten un proceso de empoderamiento. Entonces, dar vuelta la mirada al saber matemático refiere a problematizar el saber matemático (psm) de manera sistémica a partir de las cuatro dimensiones del saber: social, didáctica, epistemológica y cognitiva; para posteriormente, junto con los docentes, problematizar la matemática escolar (pme). Por tanto, replantearnos una transformación educativa respecto al desarrollo profesional docente amerita, a nuestro criterio, ampliar su mirada al saber matemático y, en particular, la matemática escolar puesta en juego en el aula.

\section{¿Cuáles son los vínculos entre empoderamiento y saber matemático?}

El empoderamiento es un fenómeno de carácter típicamente social que se ha estudiado en diversas disciplinas, por ejemplo, desde un enfoque psicosocial (Martín Mauri, 2011), social (Silva Dreyer y Martínez Guzmán, 2007), feminista (Camacho, 2003), desde la Psicología Comunitaria (Montero, 2006), o bien, desde un enfoque educativo (Howe \& Stubbs, 1998, 2003; Stolk, de 
Revista de la Escuela de Ciencias de la Educación, año 12, nRo. 11, vol. 2, Julio a diciembre de 2016. Páginas 155-176. ISSN 1851-6297. ISSN 2362-3349 (EN LINEA). LA PRÁCTICA DOCENTE MÁS ALLÁ DE LA DIDÁCTICA. DANIELA REYES-GASPERINI - RicARDo CANTORA

Jong, Bulte \& Pilot, 2011). Si bien cada una de las disciplinas tiene una mirada particular hacia el fenómeno, todas coinciden en sus características principales que nosotros hemos sintetizado de la siguiente manera: se entiende como un proceso del individuo en colectivo (es necesaria la interacción en trabajo colectivo), que parte de la reflexión para consolidarse en la acción, que se produce desde el individuo sin la posibilidad de ser otorgado (el trabajo colaborativo será necesario pero no suficiente para garantizar el empoderamiento) y, por sobre todas las cosas, transforma la realidad del individuo y su contexto.

En particular, los proyectos que tienen como objetivo impulsar el empoderamiento docente (Howe \& Stubbs, 1998, 2003; Stolk, de Jong, Bulte \& Pilot, 2011) se focalizan en darle al docente herramientas para que realicen nuevas situaciones para el aula poniendo como punto importante la contextualización, ya sea mediante el conocimiento (conocer que existe) de nuevas investigaciones relacionadas con el tema a abordar, como así también, mediante la muestra de situaciones que brinden un contexto a lo que ellos ya conocen. Todo con el objetivo de que obtengan una actitud de liderazgo, confianza y mejora en sus prácticas para la enseñanza, enfatizando el hecho de que adquieran el poder de tomar las riendas de su propio crecimiento. Si bien nosotros coincidimos plenamente con los resultados que se esperan, consideramos que este tipo de análisis se reduce a una interpretación pedagógica.

Nuestra propuesta, dado el carácter socioepistemológico que se añade a este fenómeno social, incorpora las nociones de problematización del saber matemático (psm) y problematización de la matemática escolar (pme) como base para impulsar el empoderamiento docente. Ahora bien, ¿por qué diferenciamos psm y pme? La psm refiere al hecho de "hacer del saber un problema", un objeto de análisis didáctico, localizando y analizando su uso y su razón de ser, es decir, refiere al estudio de la naturaleza de dicho saber matemático, por ejemplo relacionado con la proporcionalidad, sobre la base de preguntas del tipo: ¿qué problema llegaron a resolver las proporciones que no se podían resolver sin ellas? ¿Son más difíciles los problemas cuando las magnitudes son heterogéneas que cuando son homogéneas? ¿Por qué se trabajan problemas del cuarto valor faltante si allí no se representa la comparación, ni la construcción de una unidad de medida entre magnitudes? ¿Dónde aparecen las proporciones en la civilización? ¿Qué caracteriza a la relación de proporcionalidad? Entre muchas otras. El estudio socioepistemológico fundamentado en las dimensiones didáctica, epistemológica, social y cognitiva del saber permite confeccionar una unidad de análisis socioepistémica (uase) que provoca una singular simbiosis entre y desde las cuatro dimensiones, con el fin de generar una estructura teórica para cuestionar el saber matemático y posteriormente, consolidar estrategias para problematizar la matemática escolar. 
Revista de la Escuela de Ciencias de la Educación, año 12, nRo. 11, vol. 2, Julio a diciembre de 2016. Páginas 155-176. ISSN 1851-6297. ISSN 2362-3349 (EN LINEA). LA PRÁCTICA DOCENTE MÁS ALLÁ DE LA DIDÁCTICA. DANIELA REYES-GASPERINI - RICARDO CANTORA

En cambio, cuando trabajamos con la pme partimos de los conocimientos que para el sistema educativo son fundamentales dependiendo el nivel donde se realice el estudio. Con base en la uase se diseña una guía de actividades que confronte a las actividades didácticas típicas con el fin de poner en situación de aprendizaje al profesor y de esta manera generar espacios para que se realice la pme (Reyes-Gasperini, Cantoral y Montiel, 2014). Entendemos la pme como la acción que parte de la introspección, la mirada del que aprende y los usos que este saber posee en la cotidianeidad, apoyándose en las discusiones y reflexiones colectivas y en las investigaciones sobre dicho saber, o bien, siendo ellos mismos quienes se adentre a tal investigación. En particular, trabajar tareas donde se deban comparar magnitudes con el fin de construir una unidad de medida con la cual se permita conmensurar. Debemos retomar la idea de la constitución de proporciones como relaciones y no como una cuestión aritmética: pasar de lo aritmético a lo variacional, pues en este último, en la relación de magnitudes, se pone en juego lo proporcional.

Es necesario mencionar a esta altura que la Teoría Socioepistemológica descansa en cuatro principios fundamentales (Cantoral, 2011, 2013a): el principio normativo de la práctica social, el principio de la racionalidad contextualizada, el principio del relativismo epistemológico y el principio de resignificación progresiva o apropiación. Estos cuatro principios subyacen a la pme, pues esta problematización le permitirá al docente considerar que las prácticas sociales están en los cimientos de la construcción del conocimiento (normatividad de las prácticas sociales), y que el contexto determinará el tipo de racionalidad con la cual un individuo o grupo -como miembro de una cultura- construye conocimiento en tanto lo signifique y ponga en uso (racionalidad contextualizada). Una vez que este conocimiento es puesto en uso, es decir, se consolida como un saber, su validez será relativa al individuo o al grupo, ya que de ellos emergió su construcción y sus respectivas argumentaciones, lo cual dota a ese saber de un relativismo epistemológico. Así, a causa de la propia evolución de la vida del individuo o grupo y su interacción con los diversos contextos, se resignificarán esos saberes enriqueciéndolos de nuevos significados hasta el momento construidos (resignificación progresiva).

Por tanto, los vínculos entre empoderamiento y saber matemático están dados por la articulación del fenómeno típicamente social con el carácter socioepistemológico que subyace a su acción principal: la pme.

\section{psm y psme: El caso de la proporcionalidad}

La psm será la primera actividad por parte de los investigadores para construir las herramientas didácticas que se trabajarán a fin de realizar la pme conjuntamente con los profesores. En la uase, la dimensión social permite analizar los conocimientos matemáticos identificando la dimensión funcional, situacional e histórica, basada en la praxis, que está al nivel de la actividad 
Revista de la Escuela de Ciencias de la Educación, año 12, nRo. 11, vol. 2, Julio a diciembre de 2016. Páginas 155-176. ISSN 1851-6297. ISSN 2362-3349 (EN LINEA). LA PRÁCTICA DOCENTE MÁS ALLÁ DE LA DIDÁCTICA. DANIELA REYES-GASPERINI - Ricardo CANTORA

y que es soslayada por el $d M E$. La dimensión social, aunada a la dimensión epistemológica que estudia la naturaleza del saber, reconoce a la matemática como parte de una cultura producto de la actividad humana. Asimismo, bajo la mirada socioepistemológica, se concibe que los conocimientos se dotan de significados a través de su uso y su funcionalidad, por tanto se plantea la necesidad de que docentes y estudiantes, aunque inmersos en un sistema educativo, se relacionen con el saber matemático de una manera más activa con la intención de que construyan ideas fundamentales sobre dicho saber, más allá de las abstracciones, procedimientos y el aprendizaje propias de su aplicación. Es decir, la significación que construirá a partir de la actividad de relacionarse con el saber matemático (como aquel que es producto de la cultura), le permitirá entender aquellas nociones que las miradas platónicas consideran como "la matemática escolar". Para poder hacer este análisis, el estudio de la dimensión didáctica (1) del saber juega un papel central, pues será a través del estudio de libros de textos, programas de formación continua, notas y observaciones de clase, entre otros, que se podrá investigar cómo se manifiesta el discurso Matemático Escolar.

Asimismo, será a través del estudio de las prácticas en la construcción de casas, de la siembra, los estudios antropométricos, de la estimación de personas, que se estudiará cómo vive y se construye la proporcionalidad. ¿Cuáles son las prácticas asociadas a todas estas situaciones? La idea de comparar, aproximar, igualar, medir, son cuestiones fundamentales para desarrollar el pensamiento proporcional, distinto a la aplicación de la regla de tres simple (algoritmo aritmético). Conjuntamente con estos análisis, es a través del estudio de la dimensión cognitiva que se exploran los procesos de apropiación del saber matemático basado en el reconocimiento de que el paso del conocimiento al saber responde a procesos propios del desarrollo del pensamiento matemático, pues para el programa socioepistemológico, "la cognición es interpretada como la capacidad de hacer emerger el significado a partir de retroalimentaciones sucesivas de las acciones que las personas realizan sobre su medio ambiente próximo (tanto físico como cultural) y las interacciones de naturaleza dialéctica entre los actores" (Cantoral, 2013a, p. 61).

La construcción de la uase referente a la proporcionalidad nos ha llevado a estudiar, por ejemplo, la teoría de Piaget (1958, citado en Noelting, 1980; Inhelder \& Piaget, 1972) en donde se considera a la proporcionalidad como el sello distintivo en el desarrollo de las operaciones formales, por tanto, dado que Piaget es un referente indiscutible dentro de la educación, nosotros nos preguntamos, ¿los estudiantes (o los profesores en nuestro caso), han desarrollado este tipo de pensamiento? La idea de pasar de una relación aditiva a una relación multiplicativa parece ser la idea fundamental que se ha perseguido en los estudios referentes a la proporcionalidad.

Si nos remontamos a los orígenes reportados de la proporcionalidad, nos 
Revista de la Escuela de Ciencias de la Educación, año 12, nRo. 11, vol. 2, Julio a diciembre de 2016. Páginas 155-176. ISSN 1851-6297. ISSN 2362-3349 (EN LINEA). LA PRÁCTICA DOCENTE MÁS ALLÁ DE LA DIDÁCTICA. DANIELA REYES-GASPERINI - RICARDO CANTORA

encontramos con que si bien fue Eudoxo de Cnidos (390 A. N. E. - 337 A. N. E.), discípulo de Platón, quien trabajó con la teoría de proporciones, y se reconoce que fue Euclides quien reunió los aportes hechos por él, revisó, recapituló y transformó su teoría en su célebre Los Elementos (Guacaneme, 2012). Con el fin de comprender la proporcionalidad, hemos realizado un análisis axiomático de la teoría de proporciones abordada por Euclides en su Libro V. A la par del trabajo con Los Elementos, nos hemos puesto a trabajar con la inconmensurabilidad, por ejemplo la medida de la diagonal de un cuadrado. De allí nos surge el siguiente cuestionamiento: si no existe una medida común, ¿cómo se pueden medir estas magnitudes? El problema de medir fue sustituido, en la Teoría Euclidiana, por el problema de comparar. Esta es la pregunta fundamental que dio origen a la teoría de las proporciones entre magnitudes. Entonces, ¿será la condición provocada por la imposibilidad de medir lo que nos ha llevado a la necesidad de comparar, como la imposibilidad de adelantar el tiempo lo que nos ha llevado a predecir (Cantoral, 1990)? Es decir, ¿existe alguna práctica social subyacente? Estas preguntas son reflexiones que surgen a partir del análisis y que, de manera empírica, nos estamos acercando a su respuesta: encontrar una medida que conmensure a dos magnitudes inconmensurables es parte fundamental de lo proporcional, es decir, la constitución de la unidad de medida, que podría variar, según la referencia.

Al respecto, Guacaneme (2012) enuncia que dicha teoría emerge para atender dos problemas específicos de la época. Por un lado, ante el conflicto que había sufrido la teoría pitagórica respecto a la imposibilidad de asignar un número a la razón de dos magnitudes, los matemáticos griegos se replantearon la teoría de la proporción de tal suerte que "se pudiera hablar de razones y proporciones, sin necesidad de especificar si las magnitudes consideradas eran o no conmensurables" (Guacaneme, 2012, p. 104), en donde el mérito mayor de la teoría expuesta en el Libro $V$ es la posibilidad de comparar magnitudes inconmensurables (Corry, 1994, citado en Guacaneme, 2012). Por otro lado, Los Elementos tienen el objetivo de presentar las teorías matemáticas bajo un esquema axiomático deductivo.

Ahora bien, si la Teoría de la proporcionalidad surge a partir de la posibilidad de comparar magnitudes inconmensurables, es lógico pensar que si la mayoría de problemas que aparecen en la literatura tienen que ver con el cuarto faltante, este tipo de problemas, no requiere siempre de un razonamiento proporcional (Lamon, 1999), pues no habría nada que comparar ya que las cantidades están dadas y sobre ellas hay que operar aritméticamente, aplicando, la mayoría de las veces, la regla de tres. Asimismo, pueden hacerse enunciados con la estructura del cuarto faltante, sin siquiera existir una relación proporcional entre las magnitudes, sin embargo, los estudiantes la resolverán dado que la característica que creen suficiente para aplicar la 
Revista de la Escuela de Ciencias de la Educación, año 12, nRo. 11, vol. 2, Julio a diciembre de 2016. Páginas 155-176. ISSN 1851-6297. ISSN 2362-3349 (EN LINNEA). LA PRÁCTICA DOCENTE MÁS ALLÁ DE LA DIDÁCTICA. DANIELA REYES-GASPERINI - RICARDO CANTORA

regla de tres simple es que ambas magnitudes aumenten (contraejemplo: el precio de un taxi y los kilómetros recorridos).

Continuando la psm, ahora desde el estudio de los funcionales de Cauchy (Roa, 2010), en particular, aquel que refiere a la función de proporcionalidad: $f(x+y)=f(x)+f(y) ; x, y \geq 0 f(x+y)=f(x)+f(y) ; x, y \geq 0$ Este funcional nos ha dado luz para profundizar la diferencia entre el pensamiento aditivo y el multiplicativo. Asimismo, nos ha permitido analizar de manera profunda el ejemplo que hemos visto en la Figura 1, pues se articularon los razonamientos del modelo del pensamiento proporcional (Reyes-Gasperini, 2011; Reyes-Gasperini y Cantoral, 2014) con el funcional de Cauchy.

Retomemos la idea, ahora completa, de una de las actividades planteadas en los talleres para profesores con el fin de pme referido a la proporcionalidad y así, propiciar el proceso de empoderamiento.

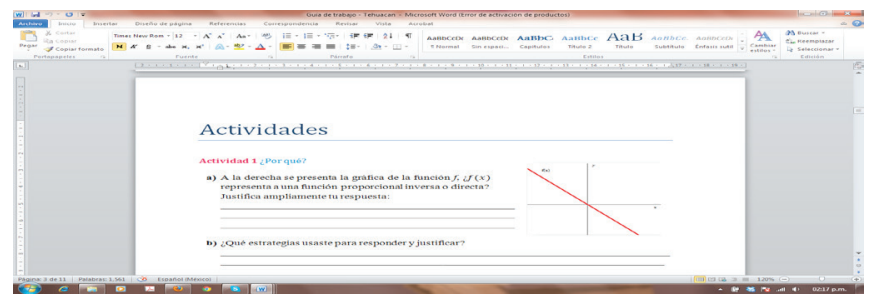

Figura 2: Actividad 1 planteada en el taller a profesores.

Más del $80 \%$ de los profesores con los que hemos trabajado contesta que esa gráfica corresponde a una función de proporcionalidad inversa, pues "a medida que aumenta $x$, disminuye $y$ ". Esta pregunta que pareciera trivial provoca una confrontación con la propia matemática escolar. Asimismo, preguntas del tipo "¿Qué significa $\frac{y}{x}=k_{x}^{\frac{y}{2}}=k$ o $y \cdot x=k y \cdot x=k$ ? ¿Qué es la proporcionalidad?", que se construyen a partir de la uase basada en la noción de proporcionalidad, de alguna manera problematizan la matemática escolar, pues le hacen preguntas a las propias expresiones algebraicas y gráficas que aparecen en libros de texto y programas escolares.

Una argumentación posible para justificar que la gráfica corresponde a una función de proporcionalidad directa es que es una recta que pasa por el origen, siendo ésta, si se quiere, otro tipo de regla nemotécnica que, por sí sola, carece de argumentaciones.

Otra argumentación que podría darse, retomando el funcional de Cauchy $f(x+y)=f(x)+f(y) f(x+y)=f(x)+f(y)$ es la siguiente: 
Revista de la Escuela de Ciencias de la Educación, año 12, nRo. 11, vol. 2, Julio a diciembre de 2016. Páginas 155-176. ISSN 1851-6297. ISSN 2362-3349 (EN LINEA). LA PRÁCTICA DOCENTE MÁS ALLÁ DE LA DIDÁCTICA. DANIELA REYES-GASPERINI - RicARDo CANTORA

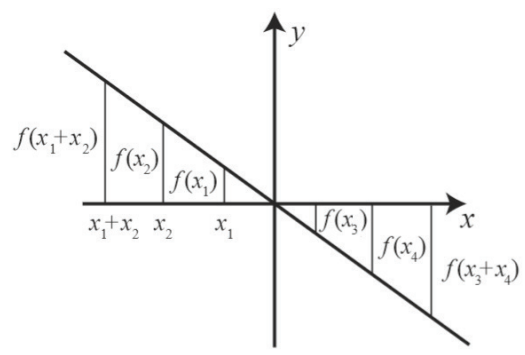

Figura 3: Argumentación visual con base en el funcional de Cauchy, que representa el razonamiento aditivo compuesto.

Asimismo, otras argumentaciones que se basan en los razonamientos del modelo del pensamiento proporcional son las siguientes:

Razonamiento aditivo simple:
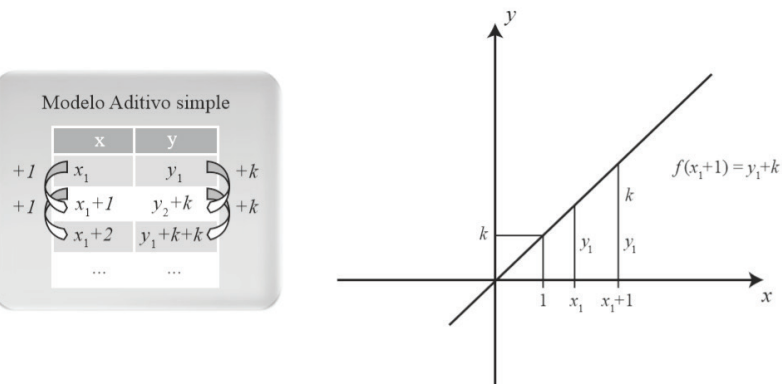

Figura 4: Razonamiento aditivo simple. 
Revista de la Escuela de Ciencias de la Educación, año 12, nRo. 11, vol. 2, Julio a diciembre de 2016. Páginas 155-176. ISSN 1851-6297. ISSN 2362-3349 (EN LINEA). LA PRÁCTICA DOCENTE MÁS ALLÁ DE LA DIDÁCTICA. DANIELA REYES-GASPERIN
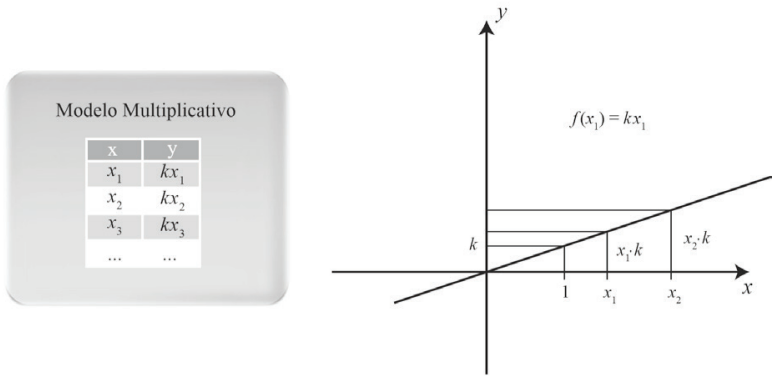

Figura 5: Razonamiento multiplicativo.

\section{Razonamiento Inter:}
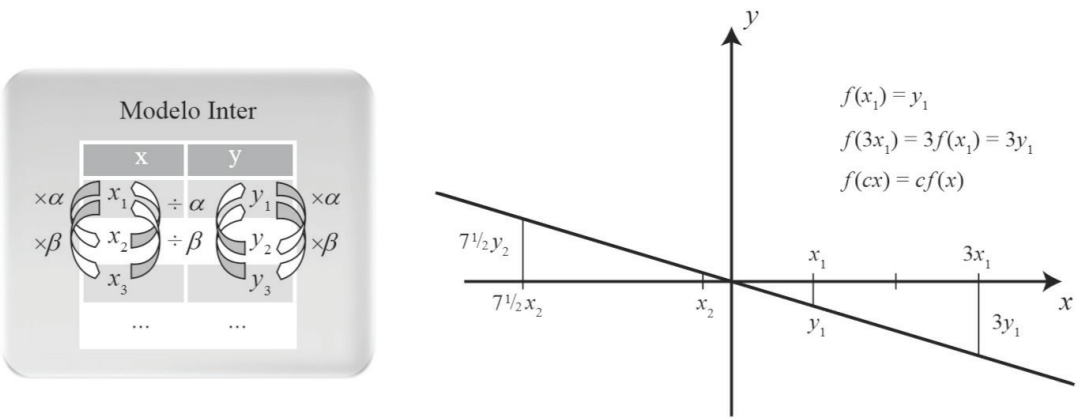

Figura 6: Razonamiento inter.

Es decir, si el desarrollo del pensamiento proporcional pasa por los distintos razonamientos hasta llegar al intra que veremos a continuación, cualquiera de las argumentaciones anteriores, ya sea en la tabla de valores o en la gráfica, podrían ser válidas para justificar por qué la función planteada es de proporcionalidad directa. Sin embargo, la normatividad del $d M E$ provoca una respuesta matemáticamente errónea (a más-menos, entonces inversa), o bien, una respuesta memorística que, repetimos, por sí sola, carece de argumentaciones matemáticas (una recta que pasa por el origen).

Ahora bien, conjeturamos que el último de los pensamientos que radica en la idea de la razón entre las magnitudes resulta ser evidente si se estudia a través de la idea de un proceso variacional más que como una relación aritmética entre dos valores.

Analicemos por qué. En la gráfica no se puede observar a simple vista la 
Revista de la Escuela de Ciencias de la Educación, año 12, nRo. 11, vol. 2, Julio a diciembre de 2016. Páginas 155-176. ISSN 1851-6297. ISSN 2362-3349 (EN LINEA). LA PRÁCTICA DOCENTE MÁS ALLÁ DE LA DIDÁCTICA. DANIELA REYES-GASPERINI - RICARDO CANTORA

relación entre las magnitudes. Sin embargo, es sabido que en las funciones de proporcionalidad directa $y=k x y=k x$, la constante de proporcionalidad $k$, suele trabajarse como un simil con su pendiente: $k=\frac{y_{2}-y_{1}}{x_{2}-x_{1}} k=\frac{y_{2}-y_{1}}{x_{2}-x_{1}}$.
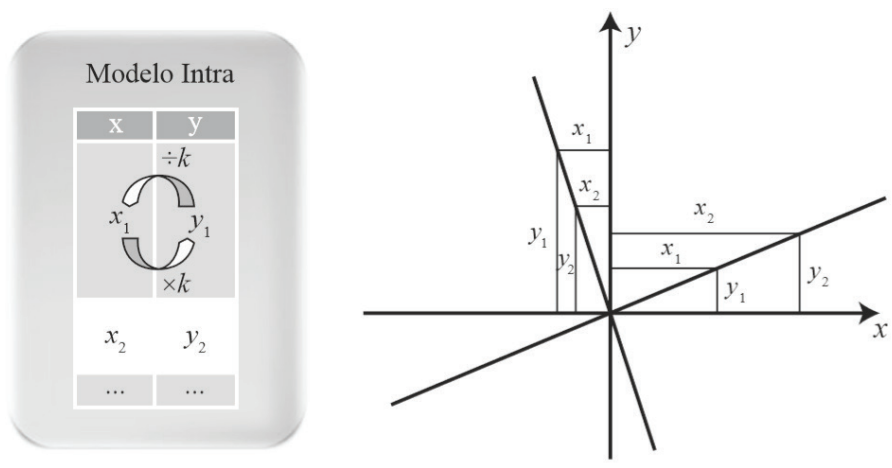

Figura 7: Razonamiento intra.

Este último razonamiento es conflictual en tanto, puede expresarse, como se hace en el programa del ciclo escolar de secundaria de México, de la siguiente manera:

\section{Razonamiento proporcional}

El razonamiento proporcional en las matemáticas.

La noción de razón surge al comparar dos números o magnitudes a través de su cociente, mientras que las proporciones resultan de comparar los valores de dos listas de números o cantidades variables para ver si guardan siempre la misma razón entre sí. Si llamamos a y b a dos cantidades, su razón está dada por el cociente:

$$
\frac{a}{b}
$$

Y si denotamos por $x$ los valores que puede tomar una cantidad variable $y$ por $y$ los valores correspondientes de la otra, decir que $x$ e $y$ son proporcionales significa que las dos cantidades están relacionadas por una expresión como la siguiente:

$$
\frac{y}{x}=k_{x}^{\underline{y}}=k \text {, donde } k \text { es la constante. }
$$

A pesar del aspecto tan sencillo de las fórmulas anteriores, las nociones de proporcionalidad y sus consecuencias son centrales en todas las matemáticas (Secretaría de Educación Pública, 2004, p. 88). 
Revista de la Escuela de Ciencias de la Educación, año 12, nRo. 11, vol. 2, JUlio a diciembre de 2016. Páginas 155-176. ISSN 1851-6297. ISSN 2362-3349 (EN LINNEA). LA PRÁCTICA DOCENTE MÁS ALLÁ DE LA DIDÁCTICA. DANIELA REYES-GASPERINI - Ricardo CANTORA

Al respecto nosotros nos preguntamos, ¿cuán sencillas serán para el estudiante estas fórmulas? Pues, demos el ejemplo de la siguiente tabla de valores, con valores sencillos, y "apliquemos" la fórmula planteada:

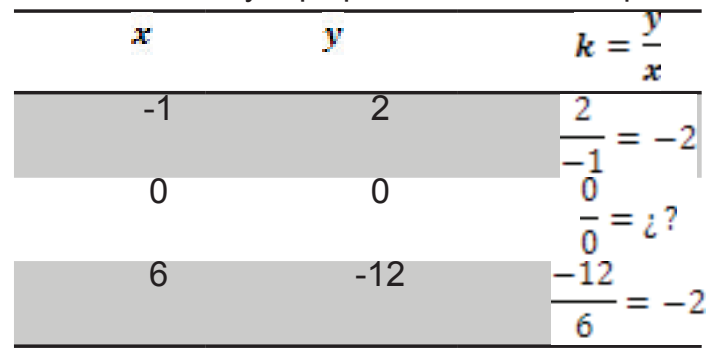

El hecho de que esa fórmula pueda ser aplicada es que subyace en ella un proceso variacional, por este motivo, es que conjeturamos que este tipo de pensamiento resulta ser evidente si se estudia a través de la idea de un proceso variacional más que como una relación aritmética entre dos valores, pues para decir que "en el caso de $\frac{00}{00}$ la constante es -2", deberíamos utilizar argumentaciones que podríamos construir, teniendo en cuenta la organización del Sistema Educativo, durante el bachiller, pues es en este momento cuando se estudia el Cálculo:

Dada $f(x)=\operatorname{axf}(x)=a x$, siendo en nuestro caso particular $a=-2$

$$
\begin{aligned}
a= & - \\
f^{\prime}(0) & =\lim _{-} \frac{f(0+h)+f(\overline{0})}{f(h)+0}=0, \\
f^{\prime}(0) & =\lim _{-} \frac{f(h+0}{h} \\
f^{\prime}(0) & =\lim _{h \rightarrow 0} \frac{-2 h+0}{h} \\
f^{\prime}(0) & =\lim _{h \rightarrow 0}(-2) \\
f^{\prime}(0) & =-2
\end{aligned}
$$

Entonces, la derivada de $f$ en cero, es la constante negativa $-2, f^{\prime}(0)=-2$ $f^{\prime}(0)=-2$. (Reyes-Gasperini, 2013).

De aquí que, conjeturamos, cuando estamos trabajando con las funciones proporcionales dentro del Cálculo matemático, ya no se están comparando magnitudes inconmensurables de donde ha surgido la idea de proporcionalidad y el desarrollo del pensamiento proporcional, sino que éste se articula con un pensamiento variacional. Por tanto, el desarrollo del pensamiento proporcional amerita instancias previas a su articulación, con el pensamiento variacional.

Entonces, comenzamos a concluir este apartado sintetizando las siguientes preguntas: ¿cuál es la naturaleza de la proporcionalidad? ¿No será que el 
Revista de la Escuela de Ciencias de la Educación, año 12, nRo. 11, vol. 2, Julio a diciembre de 2016. Páginas 155-176. ISSN 1851-6297. ISSN 2362-3349 (EN LíNEA). LA PRÁCTICA DOCENTE MÁS ALLÁ DE LA DIDÁCTICA. DANIELA REYES-GASPERINI - RICARDO CANTORA

pensamiento humano tiene una estructura aditiva más que multiplicativa y el esfuerzo realizado por los individuos no es más que poner en funcionalidad sus herramientas tanto cognitivas, sociales como contextuales? ¿Qué se trabaja cuando se trabaja la noción de la proporcionalidad en la escuela? ¿Cuáles son las dificultades didácticas -en su formación- o bien, epistemológicas, que provocan que los individuos tengan este tipo de respuestas? ¿Cómo se podría rescatar la naturaleza del saber en situaciones de aprendizaje? ¿Qué rol jugarían estas situaciones en el aula? Como dijimos párrafos arriba ¿será la condición provocada por la imposibilidad de medir lo que nos ha llevado a la necesidad de comparar, como la imposibilidad de adelantar el tiempo lo que nos ha llevado a predecir (Cantoral, 1990)? Es decir, ¿existe alguna práctica social subyacente?

Dado el análisis realizado, podemos conjeturar que la proporcionalidad tiene un origen epistemológico como estrategia para atender a la imposibilidad de medir. Asimismo, con base en los estudios realizados a partir de una propiedad matemática $((x+y)=f(x)+f(y))(x+y)=f(x)+f(y))$, se conoce que se construye la función proporcional. En términos de Vergnaud (1990), en el primer caso se precisa de estructuras multiplicativas (aquellas que requieren una multiplicación, división o combinación de ellas), mientras que en el segundo, de estructuras aditivas (aquellas que precisan una adición, sustracción o combinación de ellas). Hasta el momento, podemos postular que el pensamiento humano tiene una estructura aditiva más que multiplicativa y es por esto que en situaciones particulares en donde no se ponga en juego la comparación entre magnitudes, sino se solicite encontrar algún valor faltante, es decir, se enfatice en la cantidad más que en la relación, las respuestas llevarán a realizar procedimientos aditivos por encima de la comparación, y por tanto, por encima de la razón. Entonces, cabe estudiar qué tipo de actividades se trabajan a nivel escolar para detectar si son éstas las que obstaculizan el desarrollo deseado del pensamiento matemático escolar, pues están centradas en algoritmos para dar respuesta a preguntas concretas, más que en el desarrollo de un pensamiento proporcional que permita analizar y dar solución a problemáticas de estudio: comparar, igualar, medir.

Como se puede observar, la pregunta presentada en las Figuras 1 y 2, puede provocar la pme, en tanto confronta la forma tradicional de abordar los contenidos disponibles en el sistema didáctico con lo que le es propio al saber en juego. Pone en evidencia que aquello que se debe dominar de la proporcionalidad es insuficiente para analizar una situación de proporcionalidad. Actualmente estamos estudiando aspectos físicos, químicos, antropométricos, de construcción de viviendas, de siembra, de trueque, de Derecho, entre otros, para dar evidencia de la norma que subyace a la funcionalidad de la 
Revista de la Escuela de Ciencias de la Educación, año 12, nRo. 11, vol. 2, Julio a diciembre de 2016. Páginas 155-176. ISSN 1851-6297. ISSN 2362-3349 (EN LINEA). LA PRÁCTICA DOCENTE MÁS ALLÁ DE LA DIDÁCTICA. DANIELA REYES-GASPERINI - Ricardo CANTORA

proporcionalidad como relación adecuada. La matemática escolar es concebida como un saber institucional intocable por y para el docente, sólo suelen aceptarse modificaciones en un proceso de reforma porque provienen de las instancias oficiales de educación. Sin embargo, con el tiempo se ha evidenciado que dichas modificaciones son, básicamente, a la cantidad de contenidos y al cómo son enseñados, no al qué se enseña con estos contenidos y estas formas pedagógicas.

En ese sentido es que reconocemos que cambios en la relación del docente con el conocimiento matemático escolar generan cambios en la práctica docente.

\section{Reflexiones finales}

Para hablar de las conclusiones, inevitablemente, recordemos las preguntas que nos hicimos al comienzo del capítulo: ¿Por qué empoderar al docente? ¿En qué sentido empoderarlo? ¿Cuáles son los vínculos entre el empoderamiento y el saber matemático? ¿Cómo sabremos que está inmerso en un proceso de empoderamiento?

Con la propuesta del empoderamiento docente estudiamos el desarrollo profesional docente desde la psm y despersonificamos el problema de investigación: no buscamos causas o responsables del fracaso, sino que ponemos el foco en el papel del propio saber matemático tanto al nivel de su significado como de su uso, y desde allí proporcionamos una alternativa viable. Nuestra propuesta entonces, no provee de manera explícita estrategias didácticas o reflexiones pedagógicas sobre la enseñanza de la matemática; sino más bien, hace del saber matemático escolar un problema y enmarca sobre él un conjunto de cuestionamientos que se trabajan con los docentes, quienes localizan y analizan los usos y razón de ser del saber matemático (en este caso de lo proporcional), con base en la uase construida.

Un docente que cuestione y analice los fundamentos y procesos matemáticos de donde se derivan los algoritmos, reconozca los diversos desarrollos del pensamiento que subyacen a su construcción, es decir, las distintas formas de argumentación, e incorpore la noción de aula extendida en tanto privilegie la vida misma del que aprende favoreciendo la aparición de diversas racionalidades contextualizadas y, así, el saber adquiera un estatus funcional, y sobre todas las cosas, que cambie su relación al conocimiento matemático, es un docente que está inmerso en un proceso de empoderamiento. ¿Qué significa "cambiar su relación al conocimiento matemático"? Que transite de una perspectiva platónica, centrada en objetos abstractos ajenos a la realidad, hacia una visión socioepistemológica, bajo la concepción de que las prácticas sociales son aquellas que norman intangiblemente la forma de construir conocimiento de los individuos en grupo, es decir, nos inducen a ha- 
Revista de la Escuela de Ciencias de la Educación, año 12, nRo. 11, vol. 2, Julio a diciembre de 2016. Páginas 155-176. ISSN 1851-6297. ISSN 2362-3349 (EN LINEA). LA PRÁCTICA DOCENTE MÁS ALLÁ DE LA DIDÁCTICA. DANIELA REYES-GASPERINI - RICARDO CANTORA

cer lo que hacemos, y por tanto están en la base misma de toda construcción del conocimiento, mostrando como natural que en mayor o menor tiempo y medida, todos los individuos construyamos las nociones matemáticas basándonos en lo que en sus orígenes motivó su construcción.

Aunado a lo anterior, las actitudes de liderazgo que un docente desarrolle pasan a ser una parte importante de nuestra propuesta, pues el empoderamiento se compone de ambos elementos: problematización y apropiación del saber más la actitud de liderazgo que le permita innovar en su práctica docente, en tanto transformar su relación al conocimiento matemático. No puede haber el uno sin el otro: cuando nos refiramos al empoderamiento, no podemos hablar de la problematización de la matemática escolar sin las actitudes de liderazgo, ni bastarían las actitudes de liderazgo si no hay una verdadera apropiación del saber. Este hecho queda evidenciado en la incorporación de las interacciones con los estudiantes de manera autónoma, ya que cuando dialogamos con los docentes en los procesos de intervención, en ningún momento trabajamos la idea de llevarlo a la práctica, sino que esto surge como iniciativa propia. Aquí, es cuando el docente se vuelve agente activo.

La complementariedad entre saber matemático funcional, el constructo teórico de empoderamiento docente nos permiten postular una nueva manera de abordar la problemática de la profesionalización docente y así, atender a la exclusión que provoca el $d M E$ en los agentes del sistema educativo (Soto, 2010).

Por tanto, postulamos al empoderamiento como el proceso vivido por el docente, en conjunto con sus colegas (profesores e investigadores) con objeto de comprender, aceptar y participar de una propuesta novedosa sobre el aprendizaje centrado en prácticas y no en objetos abstractos. Ejemplo de ello, son profesores que incorporan, por ejemplo, las mezclas de colores en arte para hablar de proporciones: el color secundario es el invariante constante entre dos colores primarios. Incorporar nociones como aproximar, comparar, equivaler, medir, permitirán la significación mediante el uso del concepto de proporcionalidad directa, buscando mantener una relación adecuada entre las magnitudes que se comparan, por encima de encontrar un valor numérico per se que responde a la pregunta ¿cuánto?

Entonces, luego de haber transitado, por una síntesis del camino recorrido en el cual se explicó teóricamente aquello que la empiria nos dejaba ver, hemos caracterizado un fenómeno que de la propia realidad áulica ha emergido. Ahora podemos decir que hemos dado un nuevo paso en el objetivo de la democratización del aprendizaje, pues hemos postulado un mecanismo para estudiar al desarrollo profesional docente desde el propio saber matemático: el empoderamiento docente.

Nuestro próximo desafío será sistematizar los elementos a tener en cuenta en un diseño de intervención de desarrollo profesional docente que privile- 
Revista de la Escuela de Ciencias de la Educación, año 12, nRo. 11, vol. 2, JUlio a diciembre de 2016. Páginas 155-176. ISSN 1851-6297. ISSN 2362-3349 (EN LINEA). LA PRÁCTICA DOCENTE MÁS ALLÁ DE LA DIDÁCTICA. DANIELA REYES-GASPERINI - RICARDO CANTORA

gie dicho empoderamiento. Para ello profundizaremos en la problematización del saber matemático con el fin de evidenciar la práctica social que permanece en el ámbito normativo humano y desde allí, continuar con la problematización de la matemática escolar, pues estamos convencidos de que el saber juega un papel fundamental en la transformación educativa.

\section{Notas Bibliográficas}

(1) Lo didáctico en este enfoque, no habrá de restringirse al ámbito escolar, pues se utiliza en un sentido extendido: como acción de construcción de significados compartidos, como acto de enseñanza. La dimensión didáctica está presente en toda clase de actividades humanas, escolares y no escolares, cuando se pretende enseñar ya sea en la escuela, en los oficios, en las tradiciones populares o en las prácticas (Cantoral, 2013a).

\section{Referencias bibliográficas}

- Alanís, J. (1996). Estudio para el rediseño del discurso didáctico del cálculo en las escuelas de ingeniería: Instalación y desarrollo de un lenguaje variacional (Tesis inédita de doctorado). D.F, México: Centro de Investigación y Estudios Avanzados del IPN.

- Arrieta, J. (2003). Las prácticas de modelación como proceso de matematización en el aula. (Tesis inédita de doctorado). D.F, México: Centro de Investigación y Estudios Avanzados del IPN.

- Buendía, G. (2004). Una epistemología de los aspectos periódicos de la función en un marco de prácticas sociales. (Tesis inédita de doctorado). D.F, México: Centro de Investigación y Estudios Avanzados del IPN.

- Buendía, G. \& Cordero, F. (2005). Prediction and the Periodical Aspect as Generators of Knowledge in a Social Practice Framework: A Socioepistemological Study. Educational Studies in Mathematics, 58(3), 299-333. doi: 10.1007/s10649005-2295-5

- Caballero, M. (2012). Un estudio de las dificultades en el desarrollo del lenguaje y pensamiento variacional en profesores de bachillerato (Tesis inédita de maestría). D.F, México: Centro de Investigación y Estudios Avanzados del IPN.

- Camacho de la O., A. L. (2003). Reflexiones preliminares en torno al empoderamiento. Recuperado el 20 de noviembre de 2010 de http://www.alforja.or.cr/centros/cep/documentos/poderyempoderamiento.pdf.

- Cantoral, R. (1990). Categorías Relativas a la apropiación de una base de significaciones para conceptos y procesos matemáticos de la Teoría elemental de las Funciones Analíticas. Simbiosis y Predación entre las nociones de "el Prædiciere" y "lo Analítico". Tesis Doctoral. México: Departamento de Matemática Educativa - Cinvestav. 
Revista de la Escuela de Ciencias de la Educación, año 12, nRo. 11, vol. 2, Julio a diciembre de 2016. Páginas 155-176. ISSN 1851-6297. ISSN 2362-3349 (EN LINEA). LA PRÁCTICA DOCENTE MÁS ALLÁ DE LA DIDÁCTICA. DANIELA REYES-GASPERINI - RICARDo CANTORA

- Cantoral, R. (2003). La aproximación socioepistemológica a la investigación en matemática educativa: una mirada emergente [CD-ROM]. XI Conferencia Interamericana de Educação Matemática (tema Educación Matemática \& Desafíos y Perspectivas). Blumenau, Brazil: Universidad Regional de Blumenau.

- Cantoral, R. (2011). Fundamentos y Métodos de la Socioepistemología. Simposio en Matemática Educativa, 22 - 26 agosto 2011. D. F., México: Centro de Investigación en Ciencia Aplicada y Tecnología Avanzada del IPN.

- Cantoral, R. (2013a). Teoría Socioepistemológica de la Matemática Educativa. Estudios sobre construcción social del conocimiento. Barcelona, España: Gedisa.

- Cantoral, R. (2013b). Desarrollo del pensamiento y lenguaje variacional (Vol. 3). En R. Cantoral (Coord.). D.F., México: Secretaría de Educación Pública. ISBN: 978-607-9362-03-4. Disponible en: http://www.sems.gob.mx/work/models/sems/ Resource/6586/1/images/desarrollo_del_pensamiento_y_leng_v_smc_baja.pdf

- Cantoral, R. \& Reyes-Gasperini, D. (2012). Matemáticas y Práctica social: Construcción social del conocimiento matemático. Novedades educativas, 261, 60-65.

- Cantoral, R. \& Montiel, G. (2003a). Una presentación visual del polinomio de Lagrange. Números, 55, $3-22$.

- Cantoral, R. \& Montiel, G. (2003b). Visualización y polinomios de interpolación. Enseñanza de la Matemática, 11(1), 24 - 38.

- Cordero, F., Cen Che, C. \& Suárez, L. (2010). Los funcionamientos y formas de las gráficas en los libros de texto: una práctica institucional en el bachillerato. Revista Latinoamericana de Investigación en Matemática Educativa, 13(2), 187-214.

- Farfán, R. (2013). Lenguaje gráfico de funciones. Elementos de Precálculo (Vol. 1). En R. Cantoral (Coord.). D.F., México: Secretaría de Educación Pública. ISBN: 978-607-9362-04-1. Disponible en: http://www.sems.gob.mx/work/models/sems/ Resource/6586/1/images/lenguaje_grafico_de_funciones_baja.pdf

- Ferrari, M. (2010). Una socioepistemología de lo logarítmico. Revista Latinoamericana de Investigación en Matemática Educativa, 13(4), Número Especial, Tomo I, 53-68.

- Ferrari, M. \& Farfán, R. M. (2008). Un estudio socioepistemológico de lo logarítmico: la construcción de una red de modelos. Revista Latinoamericana de Investigación en Matemática Educativa, 11(3), 309-354.

- Godino, J. D. \& Batanero, C. (2002). Proporcionalidad y su didáctica para maestros. Granada, España: Proyecto de Investigación y Desarrollo del Ministerio de Ciencia y Tecnología.

- Guacaneme, É. A. (2012). Significados de los conceptos de razón y proporción en el Libro V de los Elementos. En O.L. León (Ed.), Pensamiento, epistemología y lenguaje matemático (pp. 99-135). Bogotá, Colombia: Fondo de Publicaciones Universidad Distrital Francisco José de Caldas.

- Hart, K. (1988). Ratio and Proportion. In J. Hiebert \& M. Behr (Eds.), Number 
Revista de la Escuela de Ciencias de la Educación, año 12, nRo. 11, vol. 2, Julio a diciembre de 2016. Páginas 155-176. ISSN 1851-6297. ISSN 2362-3349 (EN LINEA). LA PRÁCTICA DOCENTE MÁS ALLÁ DE LA DIDÁCTICA. DANIELA REYES-GASPERINI - Ricardo CANTORA

concepts and operations in the middle grades (pp.198-219). Reston, VA: National Council of Teachers of Mathematics.

- Howe, A. C. \& Stubbs, H. S. (1998). Empowering Science Teachers: A Model for Professional Development. Journal of Science Teacher Education, 8(3), 167-182.

- Howe, A. C. \& Stubbs, H. S. (2003). From Science Teacher to Teacher Leader: Leadership Development as Meaning Making in a Community of Practice. Science Teacher Education, 87(2), 281-297.

- Inhelder, B. \& Piaget, J. (1972). "El equilibrio de la balanza". En B. Inhelder y J. Piaget (Ed.), De la lógica del niño a la lógica del adolescente. Ensayo sobre la construcción de las estructuras operatorias formales (pp. 142-155). Buenos Aires, Argentina: Paidós.

- Lamon, S. (1999). Reasoning Proportionally. In S. Lamon (Ed.), Teahcing fractions and rations for understanding (pp. 223-238). New Jersey: Lawrence Erlbaum Associates Publishers.

- Lezama, J. (2003). Un Estudio de reproducibilidad de situaciones didácticas. (Tesis doctoral no publicada). D.F, México: Centro de Investigación y Estudios Avanzados del IPN.

- Llinares, S. (2012). "Del análisis de la práctica al diseño de tareas matemáticas para la formación de maestros". En N. Planas (Coord.), Teoría, Crítica y Práctica de la Educación Matemática (pp. 99-116). Barcelona, España: GRAÓ.

- Martín Maruri, I. (2011). Empoderamiento para la innovación social. En Cibervoluntarios. org (Eds.), Innovación para el empoderamiento ciudadano a través de las TIC [Versión electrónica] (pp. 129-136). Recuperado el 20 de mayo de 2010 de http://www.mas-business.com/docs/Empoderamiento\%20ciudadano. pdf

- Montero, M. (2006). Teoría y práctica de la psicología comunitaria. La tensión entre comunidad y sociedad (3era ed.). Buenos Aires, Argentina: Paidós.

- Montiel, G. (2011). Construcción de conocimiento trigonométrico. Un estudio socioepistemológico. D.F., México: Díaz de Santos.

- Montiel, G. (2013). Desarrollo del pensamiento trigonométrico (Vol. 2). En R. Cantoral (Coord.). D.F., México: Secretaría de Educación Pública. ISBN: 978607-9362-02-7. Disponible en: http://www.sems.gob.mx/work/models/sems/ Resource/6586/1/images/desarrollo_del_pensamiento_trigonometrico_baja.pdf

- Noelting, G. (1980). The development of proportional reasoning and the ratio concept. Part I - Differentiation of stages. Educational Studies in Mathematics, 11, 217-253.

- Oliveira, I. (2009). Proporcionalidade: estratégias utilizadas na Resolução de Problemas por alunos do Ensino Fundamental no Quebec. Boletim de Educação Matemática, 22(34), 57-80.

- Ponte, J. (2012). "Estudiando el conocimiento y el desarrollo profesional del 
Revista de la Escuela de Ciencias de la Educación, año 12, nRo. 11, vol. 2, Julio a diciembre de 2016. Páginas 155-176. ISSN 1851-6297. ISSN 2362-3349 (EN LINEA). LA PRÁCTICA DOCENTE MÁS ALLÁ DE LA DIDÁCTICA. DANIELA REYES-GASPERINI - RICARDO CANTORA

profesor de matemáticas". En N. Planas (Coord.), Teoría, Crítica y Práctica de la Educación Matemática (pp. 83-98). España: GRAÓ.

- Reyes-Gasperini, D. (2011). Empoderamiento docente desde una visión Socioepistemológica: Estudio de los factores de cambio en las prácticas del profesor de matemáticas. (Tesis de maestría no publicada). D.F, México: Centro de Investigación y Estudios Avanzados del IPN.

- Reyes-Gasperini, D. (2013). La transversalidad de la proporcionalidad (Vol. 4). En R. Cantoral (Coord.). D.F., México: Secretaría de Educación Pública. ISBN: 978-607-9362-01-0. Disponible en: http://www.sems.gob.mx/work/models/sems/ Resource/6586/1/images/transversalidad_smc_baja.pdf

- Reyes-Gasperini, D. \& Cantoral, R. (2014). Socioepistemología y empoderamiento docente: acciones para un cambio educativo. Boletim de Educação Matemática, 28(48), 360-382. doi: 10.1590/1980-4415v28n48a14

- Reyes-Gasperini, D., Cantoral, R. y Montiel, G. (2013). "Profesionalización docente en Matemáticas. El empoderamiento docente: una mirada emergente". En Dolores, C., J. Hernández, J., Sosa, L., García González, M. (Eds.), Matemática Educativa: la formación de profesores (pp. 153-172). México: Díaz de Santos. ISBN: 978-84-9969-664-5

- Reyes-Gasperini, D., Cantoral, R. \& Montiel, G. (2014). 'Cuando una crece, la otra decrece'... ¿proporcionalidad inversa o directa? Premisa, 16(62), 3-15.

- Roa, A. (2010). La ecuación funcional de Cauchy $f(x+y)=f(x)+f(y)$ $f(x+y)=f(x)+f(y)$ y algunas aplicaciones (Tesis inédita de maestría). Universidad Nacional Abierta, Mérida, Colombia.

- Secretaría de Educación Pública (2004). Libro para el Maestro. Matemáticas. Educación secundaria. (2a reimpresión) (pp. 88 - 105). México: Secretaría de Educación Pública.

- Silva Dreyer, C. L. \& Martínez Guzmán, M. L. (2007). Empoderamiento, Participación y Autoconcepto de Persona Socialmente Comprometida en Adolescentes Chilenos. Revista Interamericana de Psicología/Interamerican Journal of Psychology, 41(2), 129-138.

- Soto, D. (2010). El Discurso Matemático Escolar y la Exclusión. Una Visión Socioepistemológica. (Tesis inédita de maestría). D.F, México: Centro de Investigación y Estudios Avanzados del IPN.

- Stolk, M. J.; de Jong, O.; Bulte, A. M. W. \& Pilot, A. (2011). Exploring a Framework for Professional Development in Curriculum Innovation: Empowering Teachers for Designing Context-Based Chemistry Education. Research in Science Education, 41(3), 369-388. doi: 10.1007/s11165-010- 9170-9

- Vergnaud, G. (1990). La teoría de los campos conceptuales. Recherchers en Didactiques des Mathématiques, 10(2), 133-170. 
Revista de la Escuela de Ciencias de la Educación, año 12, nRo. 11, vol. 2, JUlio a diciembre de 2016. Páginas 177-194. ISSN 1851-6297. ISSN 2362-3349 (En LiNEA). The MEANINGS OF UNIVERSITY SOCIAL COMmitMent. CARINA ANDREA GeRLeRo

\title{
THE MEANINGS OF UNIVERSITY SOCIAL COMMITMENT. AN APPROACH TOWARDS CONSTRUCTING THE STATE OF THE ART
}

\author{
Carina Andrea Gerlero* \\ Rosario National University - Ministry of Education of Santa Fe, Argentina. \\ cgerlero@hotmail.com \\ Received on: 07/04/2014 Accepted on: 11/04/2014 \\ Translated from the Spanish by \\ Agustina F. Casero \\ Body of Translators, Rosario National University (UNR), Argentina
}

\section{Resumen}

El presente artículo (1) tiene como objetivo identificar y analizar las perspectivas teóricas sobre el Compromiso Social Universitario (CSU) a partir del relevamiento del conocimiento producido en el período 2007-2013, con el propósito de precisar qué se entiende por CSU y a qué modelo de Universidad suscribe. Se indagan relaciones entre éste y la tradición reformista de las universidades públicas argentinas, principalmente en lo referente a su función social. Se analizan las similitudes y diferencias entre el CSU y la Responsabilidad Social Universitaria (RSU), tratando de discernir si se trata de una cuestión semántica o si se sostienen en diferentes valores y conceptos.

Se identifican algunos principios obligados al momento de referirse al CSU, tales como: la definición de proporcionar educación universitaria de calidad igual para todos; la democratización y la orientación solidaria de las acciones universitarias, a través de las funciones de docencia, extensión, in-

* Department of Education Planning and Articulation. Ministry of Education, Province of Santa Fe, Argentina. Specialist in High Education Policies and Management (CEIUNR). PhD candidate in Education of Experimental Sciences (FBCB - UNL). 
Revista de la Escuela de Ciencias de la Educación, año 12, nRo. 11, vol. 2, Julio a diciembre de 2016. Páginas $177-194$. ISSN 1851-6297. ISSN 2362-3349 (EN LinEA). THE MEANINGS OF UNIVERSITY SOCIAL CommitMENT. CARINA ANDREA GERLERo

vestigación y gestión; la necesidad de establecer un diálogo con la sociedad, para contribuir, conjuntamente, a desarrollar la cultura y los procesos de integración regional, disminuir la exclusión y brechas de desigualdad, profundizar la democracia y el bien común, asumir un compromiso ético con la transformación y construcción social.

\title{
Palabras Clave:
}

Universidad - Función Social - Democratización - Diálogo - Compromiso ético.

\begin{abstract}
The object of this paper is to identify and analyze the theoretical perspectives about University Social Commitment (USC) based on a survey of the knowledge produced between 2007-2013, in order to specify the meaning of USC and to which model of University it subscribes. The relationship between USC and the tradition of reformist Argentine public Universities is assessed; mainly with regard to its social function. The similarities and differences between USC and University Social Responsibility (USR) are analyzed in order to decide whether this is a semantic matter or whether these are notions based on different values and concepts.

Some significant principles are observed when referring to USC, such as: the decision to provide a quality university education equal for all; the democratization and solidarity-based orientation of university actions by means of its teaching, extension, research, and management functions; and the need to establish a dialogue with society so as to jointly contribute towards developing culture and regional integration processes, reducing exclusion and inequality gaps, deepening democracy and the common good; as well as making an ethical commitment to social transformation and construction.
\end{abstract}

\section{Keywords:}

University - Social function - Democratization - Dialogue - Ethical commitment.

\section{On the Constitution of the Field of University Social Commitment}

In Latin America, public universities are simultaneously sustained and organized on the basis of two wide models: the professionalizing University and the scientific University, which make their very own imprint as a result of the ideas derived from the 1918 Reform. Its postulates were a starting point for the processes of democratization of HEls, involvement of university agents in national political and social issues, interrelation between Latin American 
Revista de la Escuela de Ciencias de la Educación, año 12, nRo. 11, vol. 2, Julio a diciembre de 2016. Páginas 177-194. ISSN 1851-6297. ISSN 2362-3349 (EN LiNEA). The MEANINGS OF UNIVERSity SOCIAL COMMITMENT. CARINA ANDREA GeRLERO

universities and innovation in academic programs. Progressively, Reformist Universities supported open and free access, academic freedom, access to faculty positions through competitive examination, university autonomy and autarchy, participation of faculty, students and graduates in university and college government, university extension, modernization of curricula and the faculty's pedagogic strategies, among others.

Although this model of a Reformist University was settled among university agents, it did not have a continuous development throughout history. In general terms, it was fostered during democratic periods and reduced during periods with governments installed through dictatorships. From that moment to our days, during democratic periods, universities have strengthen their ties with society through different formats, models or strategies, which will only be dealt with tangentially here, since the time frame exceeds the limits of this paper.

Based on the analysis of available documents and bibliography from 2007 to 2013 , we can observe, in the last few years, the configuration of a field of specialized academic production on Higher Education (HE) in reference to USC. Between 2007/2008 there were certain events that seemed to determine the emergence of this field. In Latin America and the Caribbean, the 2008 Regional Conference on Higher Education (CRES) has clearly occupied a central place, since it defined the prospects for $\mathrm{HE}$ in the region. Views from the CRES, along with views arisen in conferences from other regions, are part of the input that came into play during the 2009 World Conference on Higher Education (WCHE) held in Paris. These perspectives have enabled an understanding of the meanings of USC and have enriched theoretical productions, debates and research, while favoring the configuration of SC as an autonomous concept, in the field of university $\mathrm{HE}$.

It is interesting to review the approaches arisen during different exchanges about university-related topics which serve as precedents for the Conferences. In this respect, we place the 1996 CRES held in La Habana and the 1998 WCHE, which took into account, among other aspects, the document prepared by UNESCO in 1995: "Policy Paper for Change and Development in Higher Education". This document resulted in the need to strengthen equity, quality, pertinence and internationalization in the field of HE. These stages showed the influence of policies typical of the '90s and, although there were views concerning the relation between University and society, there was still no structuring of them in terms of USC.

More recently, we can mention the International Conference of Latin American and Caribbean University Rectors: "THE SOCIAL RESPONSIBILITY OF LATIN AMERICAN AND CARIBBEAN UNIVERSITIES", held at the Federal University of Minas Gerais on September 2007. The declaration issued by the Rectors who took part (2007, p. 2) suggests: "Conceiving the social commit- 
Revista de la Escuela de Ciencias de la Educación, año 12, nRo. 11, vol. 2, JUlio a diciembre de 2016. Páginas 177-194. ISSN 1851-6297. ISSN 2362-3349 (EN LINEAA). The MEANINGS OF UNIVERSITY SOCIAL COMMITMENT. CARINA ANDREA GERLERO

ment of universities (...) as an implementation of institutional policies whose premise is education as a human right, a public good, which takes into consideration the social factor, in accordance with quality, pertinence, relevance, inclusion and equity values". On the other hand, they recognize that the work of the 1998 WCHE on the pertinence of HE allows considering USC in relation to the scope of an education which takes into consideration the social factor. They understand that, to achieve this purpose, it is necessary to construct a kind of knowledge that contributes to reducing those inequities strengthen by globalization processes and guarantying those rights which favor a better quality of life. They also propose different topics to be addressed by the university agenda, such as committing to expand coverage while meeting the need for quality social inclusion, leave HE out of GATS (General Agreement on Trade in Services) agreements and promote a solidarity-based internationalization of HE in Latin America and the Caribbean, among others. Nevertheless, the main prospect on the agenda lies in the issue of "promoting a qualitative leap in the social commitment of Universities, stimulating the generation of strategic changes", in a way that allows moving from poorly systematized low profile isolated actions to programmatic lines which take part in the missions of Higher Education Institutions (HEI).

Within the Conference of Rectors, we highlight Miguel Rojas Mix's presentation on "Social Commitment of Latin American and Caribbean Universities". He suggests that the root of USC is to provide quality education, on equal basis, for all citizens. He specifically refers to USC as:

(...) a broad commitment that spreads over different fields related to the reproduction and improvement of the social model: equity, science, professional efficiency, culture and identity, ideological pluralism, social ethics, preservation of historical memory and universality of knowledge, as well as the creation of a critical mass (Rojas Mix, 2007, p.179).

Moreover, he points out that the roles University must embrace to fulfill this commitment are: to develop culture and cultural integration, as historical memory forges the imaginary in the construction of identity and enables critical thinking in a globalized world; to direct its action towards the common good in service of the nation, which gives pertinence to HE, since it allows to distinguish and contextualize what is better for its culture and what strengthens its development; to teach values, develop and defend democracy, in a way that allows for a recovery of the relation between democracy -in the political field-, philosophy -in the field of knowledge- and the ability to live and think rationally in society; to reduce the gaps and inequities that threaten social justice, leaving aside the liberal tendency that promotes the substitution of democracy by the market and of the political factor by the economical factor.

Rojas Mix (2007) states that the development of USC requires the cons- 
Revista de la Escuela de Ciencias de la Educación, año 12, nRo. 11, vol. 2, Julio a diciembre de 2016. Páginas 177-194. ISSN 1851-6297. ISSN 2362-3349 (EN LiNEA). The MEANINGS OF UNIVERSity SOCIAL COMMITMENT. CARINA ANDREA GeRLERO

truction of a constant dialogue between University and different local, national and regional social agents. On this level, it is important the role of University as guarantor of the access to higher education based on equal opportunities and the teaching of values, which favors critical thinking while defending and building individual and collective rights.

The International Conference of Latin American and Caribbean University Rectors also included the presentation of Roberto Tejada Reyna, Rector of the Autonomous University of Santo Domingo. In his exposition, entitled "Proposals for an Agenda on the Social Commitment of University", Tejada Reyna (2007) stated that the mission and philosophy typical of University place it as an institution with SC. Regarding the agenda, the following issues were highlighted: the need to build it in accordance with the social agenda, since this will provide meaning and pertinence to university actions; the commitment to shape empathetic and value oriented professionals; the development of university curricula invested in improving people's quality of life; active participation in the achievement of the Millennium Development Goals established by UNESCO, especially when it pertains to the promotion of an equitable distribution of wealth and the eradication of extreme poverty, hunger, illiteracy and child mortality, among others.

On November 2007 the 9th Latin American Congress of University Extension took place in Bogotá, Colombia. The main conference, in charge of Carmen García Guadilla, entitled "Social Commitment of Universities", referred to USC as something inherent to university purposes, and analyzed the different ways of understanding and naming it, especially from the mid 20th century to the present.

Her presentation states that during the ' 50 s and ' 60 s, USC represented the developmentalist ideology in the sense that education would allow for underdeveloped countries to overcome this situation. The premise was that more education equals more productivity and social mobility. According to García Guadilla, that is the reason some authors have named this period "the age of innocence", since education alone cannot fulfill this role. She recovers Paulo Freire's view regarding the idea of university extension as a process of dialogical and horizontal communication between University and society, as opposed to the unidirectional set-up, where universities "give" and society "receives".

She places "the age of skepticism" during the "70s, highlighting the emergence of socio-educational critical theories, influenced by the reproductivism of Bordieu and Passeron as well as by the idea of Darcy Ribeiro, who proposes replacing the "traditional university" with the "necessary university", which favors University's commitment towards society. The ' 80 s are described as a "lost decade" due to the economic crises that took place at the beginning of the globalization process. She interprets that the influence of critical theories diminished while some participatory action approaches emerged, which, in 
Revista de la Escuela de Ciencias de la Educación, año 12, nRo. 11, vol. 2, JUlio a diciembre de 2016. Páginas 177-194. ISSN 1851-6297. ISSN 2362-3349 (EN LINEAA). The MEANINGS OF UNIVERSITY SOCIAL COMMITMENT. CARINA ANDREA GERLERO

the field of $\mathrm{HE}$, were associated with the production and distribution of knowledge regarding the social environment. The ' 90 s vindicate the approaches of Freire, Echavarría, Ribeiro and other Latin American referents in view of the new scientific paradigms. This period questions certainty and determinism and values social subjectivity, social agents and democracy as the "expansion of reflective consciousness in every area of social interaction". On the one hand, this enables a new understanding of the world, based on diversity, pluralism, values of solidarity, responsibility and mutual respect. On the other hand, it grants value to the production of contextualized, integrated and interdisciplinary knowledge. Nevertheless, at the same time, the neoliberal policies of $\mathrm{HE}$ are also established during this decade.

Finally, she characterizes the 21th century as the "age of social commitment", understood as a commitment to human and sustainable development in order to face globalization and its resulting inequalities. She expresses that:

Social commitment as a requirement is one of the key elements of the late 2010s. Social commitment must overcome the limitations present in the concept of extension, vindicating a necessary contextualization of knowledge, an interaction with society, shared learning processes oriented towards transdisciplinarity, increasingly viable interactive processes between education and society, a new perception of networks as collective learning organizations and an education without borders (García Guadilla, 2008, p.34).

We should stress that these meetings taking place at a regional level have converged in two broad Conferences. The Regional Conference on Higher Education in Latin American and the Caribbean, on June 2008 in Cartagena de Indias and the World Conference on Higher Education: "The New Dynamics of Higher Education and Research For Societal Change and Development", on July 2009 in Paris.

The CRES' Final Declaration (2008, p.2) expresses that the main purpose is "to establish a scenario that makes it possible to articulate in a creative and sustainable manner policies that strengthen the social commitment, quality and pertinence of higher education and the autonomy of Universities". As regards USC, the following points are highlighted:

[Its position regarding] the humanistic character of higher education (...) [and the resulting orientation] towards the comprehensive training of persons, citizens and professionals, able to approach [the needs of the context] with ethical, social and environmental responsibility (...) and who can actively, critically, and constructively participate in society.

[The fostering of] respect for and defense of human rights (...) [by including 
Revista de la Escuela de Ciencias de la Educación, año 12, nRo. 11, vol. 2, Julio a diciembre de 2016. Páginas 177-194. ISSN 1851-6297. ISSN 2362-3349 (En LiNEA). THE MEANINGS OF UNIVERSITY SOCIAL COMMITMENT. CARINA ANDREA GERLERO

them in] educational programs, as well as in the priorities of research, extension and inter-institutional cooperation. (...)

[The decision to] move forward in establishing a more active relationship [between Universities and their contexts].

[The linking of quality] to both pertinence and responsibility toward sustainable development [of society]. (...)

[The need for] an academic model marked by the examination of problems within their contexts; the production and transfer of the social value of knowledge; joint work with communities; scientific, technological, humanistic and artistic research based on an explicit definition of the problems being addressed, of fundamental interest for national or regional development, and the well-being of the population; an active dissemination effort aimed at educating for citizenship, rooted in respect for human rights and cultural diversity; extension activities that enrich education, helping to detect problems for the agenda of research, and that create areas for joint action involving diverse segments of society, especially the most neglected. (Declarations of the Regional Conference on Higher Education in Latin American and the Caribbean, 2008, p.6)

The communiqué of the 2009 WCHE also exhibits views related to USC or USR when referencing:

The social responsibility [of higher education] to advance our understanding of multifaceted issues, which involve social, economic, scientific and cultural dimensions and our ability to respond to them. (...)

Higher education must not only give solid skills for the present and future world but must also contribute to the education of ethical citizens committed to the construction of peace, the defense of human rights and the values of democracy. (...)

Member States, working in collaboration with all stakeholders, should develop policies and strategies at system and institutional levels to (...) ensure adequate investments in higher education and research to reflect growing expectations and societal needs. (Communiqué of the World Conference on Higher Education, 2009, pp. 2-8).

Contributions made by the participants present at these Conferences, Meetings and Congresses, whether made by speakers or university representatives, contributed to starting a configuration of the field of USC. Moreover as the subject was addressed by the heads of HEls, it was easier to include it in the university agenda. The fact that this happened within spaces of dialogue marks a clear position in the field of education. There is great value not only in their calling for action but in the institutional commitment taken on from different university functions towards the development of concrete policies rooted in social commitment for every country and territory affected by their actions. 
Revista de la Escuela de Ciencias de la Educación, año 12, nRo. 11, vol. 2, Julio a diciembre de 2016. Páginas $177-194$. ISSN 1851-6297. ISSN 2362-3349 (EN LinEA). THE MEANINGS OF UNIVERSITY SOCIAL CommitMENT. CARINA ANDREA GERLERo

As a result of these meetings, the view of USC was installed in the university agenda and is still present today through different efforts and actions carried out by HEls on the complex broad topics comprised in USC. This strengthens the thought that USC is a commitment inherent to University as well as a political and ideological principle affecting all university functions.

We notice that the relation between USC and the social and cultural values of education is focused on reducing the negative impacts of globalization and other neoliberal policies. Moreover, the hope is to reduce the inequalities faced by our societies through increasing citizens' participation in the decision-making process concerning issues and situations that involve them. The access of more young people to a comprehensive university education, a humanistic educational model, the development of research pertinent to social needs as well as extension practices which favor a dialogue between University and society are the basis for achieving these goals.

\section{Theoretical Approaches to University Social Commitment and University}

\section{Social Responsibility}

USC is conceived in a polysemic manner, having multiple different meanings. This section aspires to analyze the approaches and conceptions in question so as to understand the meaning attributed to USC. It could be thought that the different ways of conceiving USC are related to different ways of understanding the University, its mission and the actions that define which should be its core functions. Specifying the meaning of SC calls for mentioning the underlying view as regards the "Idea of University" and the meanings assigned to University HE.

It would be interesting to divide the concepts according to its underlying theoretical approaches and observe how other aspects of USC come into play, in an attempt to enrich the analysis. In this respect, we will, on the one hand, address the conceptions that refer to USC and USR as a strategy based on the University's social function and non-transferable mission of building the society it falls within. On the other hand, we will analyze the conceptions built from a logic near to Corporate Social Responsibility (CSR), which, as a result, link USR with the University's role of environmental impact management, assessment and accountability.

\section{Social Commitment and the Social Function of University}

This section will address perspectives that relate USC to the social role of University. Here we place the conception of Miguel Rojas Mix (2007), who expresses the need for a quality education for all that enables the education of a "social professional" committed to situations typical of a specific historical, political and social time and context (2). 
Revista de la Escuela de Ciencias de la Educación, año 12, nRo. 11, vol. 2, JUlio a diciembre de 2016. Páginas 177-194. ISSN 1851-6297. ISSN 2362-3349 (En LiNEA). The MEANINGS OF UNIVERSity SOCIAL COMMITMENT. CARINA ANDREA GeRLeRo

On a similar line of analysis, in the context of a research project on University Social Commitment (3), Cecchi, Lakonich, Perez and Rotstein (2009, pp.11-12) state that USC is the basis for discussing the objectives of University and the ways in which Latin American Universities link their actions to national and regional issues, especially those concerning the most vulnerable segments. They place the emergence of the idea of USC in Argentina during the 1918 University Reform and observe that this idea is not a constant feature in Argentina. It is present during democratic periods and absent during periods of successive military coups d'état. They identify the scientific-technological paradigm as an obstacle to USC, since it conceives academic roles dissociated from their context of development.

The authors suggest thinking USC from three principles: "Quality Education for all" (as established by UNESCO), "Purpose" and "Identity" (both suggested by Rojas Mix). Regarding the purpose of University HE, they refer to what kind of professionals should be formed, and that they support, as well as Rojas Mix, the idea of a social professional, who would act guided by the principle of solidarity. On the topic of University Identity, they propose relating it to peace, culture and the reproduction and improvement of a social model of integration. They also retrieve different contributions made by Taborda and Freire which favor the idea of USC as:

(...) a way of writing the cultural world, and that means a way of "writing it", where this writing - even when we consider thought coming from previous readings and writings, never isolated nor individual - is a creative act, an act which is not restricted to a premature closure of meaning behind experience, life and the world, but which registers the opening of other possible worlds and experiences built on the University-Society relation (Huergo, 2006, cited by Cecchi et al., 2009 , p. 41)

In turn, Boaventura de Sousa Santos (2005, p. 78) suggests that Universities must assume their URS and accept being affected by social demands, especially by those coming from groups with little power to command them. He reflects on what enables meeting the challenges of USR, i.e. autonomy, academic freedom and appropriate institutional conditions, and understanding that regional and local contextual challenges cannot be faced in a rigid or general manner.

Moreover, the author suggests that the University must regain its legitimacy through a broad USR program that includes action lines related to access, extension, research-action, the ecology of knowledge, the University-Public School relation and the University-Industry relation.

The following is a brief analysis of each line. We notice that, based on its position, SC has to do with going beyond massification in order to move towards in the democratization of access. To this end, it is necessary to enable 
Revista de la Escuela de Ciencias de la Educación, año 12, nRo. 11, vol. 2, JUlio a diciembre de 2016. Páginas 177-194.

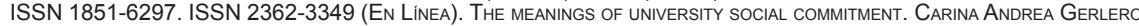

free access and financial aid (scholarships) for working-class students; the joint work between Universities and public schools to promote the integration of both systems and improve teaching and learning conditions in the fields of pedagogy and science; and positive action measures that confront discrimination associated to racial, ethnic and social vulnerability conditions. Furthermore, another central aspect proposed is the revision of spread knowledge to avoid elitist, meritocratic or privilege-based conceptions in present and future generations. Here it is important that the education of teachers be focused on the public sphere rather than on principles promoted by institutions subject to modalities of the education market.

Moreover, he notices that SC emerges whenever there are extension activities ideologically and politically based on "building social cohesion, deepening democracy, fighting social exclusion and environmental degradation, advocating for cultural diversity". (By Sousa Santos, Ibid.:67). In this line of argument, research-action is worth it as long as it contemplates and involves the participation of non-university social agents in its design and execution. The author also thinks that the ecology of knowledge deepens research-action while acting as an inverse extension: it allows for knowledge originated in different spheres - popular, layman, traditional - to engage in dialogues with the scientific and humanistic knowledge produced at University. These strategies may provoke a solidarity-based reorientation of the University-society relation.

On the other hand, he points out that the concepts of "knowledge society" or "knowledge economy" attempt to center the production of knowledge from a neoliberal paradigm so as to aid corporations, therefore increasing their profits. This conception turns knowledge into a private or privatizable good, exchangeable in the market. On the contrary, an action based on USC demands for the agenda of scientific research to be established and regulated according to multiple social needs, enabling wider research areas and exchanges between universities and companies, which do not necessarily involve an academic dependency or the financial survival of HEls.

Another contribution relevant to the topic of USC, which strengthens the idea of its connection with a social function, is the one made by Cruz Ayuso and Sasia Santos (2008), as they focus the attention of University Commitment on the construction of citizenship as well as on the power of these institutions to influence society and enable actions that can change situations of great social vulnerability. In this sense, they understand that the education of responsible citizens, the contribution to social development and the generation of new knowledge contribute to SR without being an end in themselves. These aspects enable the transformation and construction of society from the point of view of ethics and social justice. Therefore, they argue that thinking the concept of responsibility from the field of CSR and from the concepts of 
Revista de la Escuela de Ciencias de la Educación, año 12, nRo. 11, vol. 2, Julio a diciembre de 2016. Páginas $177-194$. ISSN 1851-6297. ISSN 2362-3349 (EN LinEA). THE MEANINGS OF UNIVERSITY SOCIAL CommitMENT. CARINA ANDREA GERLERO

corporate management confuses and restricts the scope of USC, since University is not supported on the principle of economic "utility", nor should its social action be reduced to aid or charity actions. They suggest thinking the concept of responsibility from the following dimensions of analysis:

A primary consequentialist meaning which, as regards the decision-making process, stresses the analysis of consequences and calls upon taking responsibility for the potential impacts derived from this process, especially the negative ones. A secondary contractual meaning which stresses the importance of everyone's responsibility - people and organizations-, as significant actors or agents of a society. Finally, a third prospective stage which highlights the a priori aspect of such duty and the need to be concern about, deal with and take responsibility over things. This stage refers to people or organizations willing to take over, through their actions or activity, the regeneration of our unequal and unfair societies (de la Cruz Ayuso \& Sasia Santos, Ibid::35).

They suggest moving forward towards a third meaning of responsibility, one that will enable an understanding of University as an institution of society and of its commitment with social matters as well as its modalities of interaction with and intervention in social issues.

We can notice that the conceptions expounded so far focus on how to involve the University, on the basis of its essential functions, in the construction and transformation of the societies they are part of. Here lies the relation between SC, social function and a model of progressive University, actively involved in contextual liaisons.

In this sense, the most relevant proposals have to do with the challenge of educating a social professional and the need for a solidarity-based reorientation of the link between University and society. An interaction that, within a framework of dialogue and articulation of policies, enables a positive transformation of living conditions for the most vulnerable segments, promotes a democratic coexistence of ideas, and values the contributions arising from social and cultural diversity. These proposals allow for an observation of how USC links itself with the principles of the 1918 University Reform as well as of the need to deepen the reach of reformist principles, adapting actions to address present challenges.

\section{University Social Responsibility from a Corporate View}

This section will review the approaches that link USR with CSR. On this line of analysis, we place the conception of Torres Pernalete and Trápaga Ortega (2010), who establish a link between the social role of University (4) and the present definition of university extension, USC and USR, among others; although they place mainly the most recent precedents of USR as a concept 
Revista de la Escuela de Ciencias de la Educación, año 12, nRo. 11, vol. 2, Julio a diciembre de 2016. Páginas $177-194$. ISSN 1851-6297. ISSN 2362-3349 (EN LinEA). THE MEANINGS OF UNIVERSITY SOCIAL CommitMENT. CARINA ANDREA GERLERo

in the late '90s, on occasion of the escalation of globalization. They suggest that the paradigm of USR emerges as a result of the need for a new social contract between University and Society, promoted by the University Network for Ethics and Social Development, of the IDB, which implemented the Support Program for Initiatives of USR, Ethics and Development in 2006. Here USR is seen as a concept formulated in terms of impact management (5), related to the fundamental functions of University: teaching, research, extension and management.

Moreover, the authors suggest that the central aspect of USR is the configuration of an ethical conduct which includes a joint responsibility with the environment. In this sense, they understand that University, besides creating professionals and providing an educational service, is an institution "serving all that is relevant for the individual and for society in general". They consider that a socially responsible University addresses its own needs as well as social needs; promotes learning to achieve sustainable human development; should worry about its own activities and member's quality of life; should adopt a critical position and a position of permanent evaluation, and should have a USR management body.

The conception of François Vallaeys, de la Cruz and Sasia (s, f) refers to USR in terms of impact management and recovers the concepts of CSR so as to apply them to USR. They define USR as "aiming to reposition University in society lining up its four processes (management, teaching, research and extension) with the mission, values and social commitment of University" (:22). They notice that USR is rooted in ethics and actions which, in practice, can make its development possible.

On the general impacts that universities should manage, they propose to encourage a "responsible campus", promoting an organization culture of University that recovers and strengthens labor, social and environmental rights while fostering human and environmental development as well as increasing social capital. In relation to University's own impacts, on the one hand, they consider those of an educational nature, which stand for the education of citizens and professionals responsible to society. On the other hand, they refer to cognitive and epistemological impacts, which tend to a social appropriation of knowledge and include the participation of non-academic agents in research practices.

They understand that USR defines and improves USC as it creates a need for management at University and provides the tools to this end. Additionally it promotes a dialogue with and participation of different university and non-university agents.

Nonetheless, Saravia and Escobar (2010) relate SR to addressing the needs of the population, to ethics and human rights, and to the challenge of contributing to common good and sustainability. They analyze USR as part 
Revista de la Escuela de Ciencias de la Educación, año 12, nRo. 11, vol. 2, Julio a diciembre de 2016. Páginas 177-194. ISSN 1851-6297. ISSN 2362-3349 (EN LiNEA). THe MEANINGS OF UNIVERSITY SOCIAL COMMITMENT. CARINA ANDREA GeRLeRO

of the SR inherent in organizations from different origins(social, political and economic) as well as in the State. They identify that the notion of SR, which was initially developed for business organizations, has extended to other spheres. They also consider that, at present, the labor markets and the globalization of communications call for knowledge, causing some differences with the previous forms of "value creation" in the market. The economic value of information, although intangible, constitutes the main generator of economic value. Therefore, they understand that social justice is related to an equitable distribution of knowledge focused in the needs of society.

Margetic and Suarez (2006) analyze the Statutes of National Universities as regards their mission, social function and university extension, so as to identify how institutional modalities of commitment with national issues present themselves. They notice that the "ought to be" present in the Statutes, generates tension between aspirations of universality and particularity, and do not always agrees with ongoing university policies. Furthermore, they acknowledge that "this attitude of paying attention to their own contexts is defined as social responsibility, sensitivity and, principally, as a solidarity-based commitment with the community" (:27).

The perspectives outlined in this section recover the need to tie University actions with its environment, in order to favor sustainable human development and social justice. Although they think of USR as a modality that enables such tie, the stress is on how the management of these impacts is carried out in HEls.

The authors express the need to implement processes and mechanisms of institutional assessment, accountability, organizational good practices and advertising of action. Thus, they understand that there is a social contract established between University and society, as well as an ethical management and organization culture built taking into consideration labor, social and environmental rights. These concepts, originated within the paradigm of CSR, can contribute to strengthening institutionality and, therefore, to achieving social goals through different functions of University. But they can also constitute an element that links its practices to market rules. Therefore, strong monitoring is required so as to prevent a blurring of the main objectives pursued by SR.

The contribution of USR lies in emphasizing the need to manage so as to transform. In order to achieve a real contribution to their objectives, university projects and actions require strategic planning. Nevertheless, the challenge is to prevent management from becoming an end in itself, denaturing its raison d'être to be established as a privileged source of authority that sets university objectives and, therefore, leading education towards a neoliberal model. 
Revista de la Escuela de Ciencias de la Educación, año 12, nRo. 11, vol. 2, Julio a diciembre de 2016. Páginas $177-194$. ISSN 1851-6297. ISSN 2362-3349 (EN LinEA). THE MEANINGS OF UNIVERSITY SOCIAL CommitMENT. CARINA ANDREA GERLERo

\section{Concluding Thoughts}

The most important contribution from the perspective of USC, as something inherent to the social function, is concerned with addressing University objectives as related to what is external to the institution. The idea is to think the objectives based on University autonomy while focusing on society. This position places citizens and the social factor first, so as to transform living conditions, favor social inclusion and build more democratic and fair societies. Although University processes do not lose their specificity, they are organized and developed so that their basic functions build upon what is specific of a discipline and upon how such discipline relates to its professional, cultural and social context. Therefore, dialogue-based contributions as well as the articulation of actions between political, social and State agents become important. This idea is founded in a humanistic and progressive model of Education and University, which, in the case of our Latin American identity, follows the ideological and political line launched by the 1918 reformists. The main challenge for these proposals is to avoid remaining a mere declaration of principles and become real in everyday practices.

The analysis on the field of USC, concerning both the events which made its emergence possible and the contributions from research and theoretical conceptions, consolidates its indissoluble link with the social function of University. Thus, we identify some essential principles when referring to USC, such as:

- The decision to provide quality University education for all, on equal basis.

- The democratization and solidarity-based orientation of University actions, through teaching, extension, research and management functions.

- The need to establish a dialogue with society, so as to jointly contribute to developing culture and regional integration processes, reducing exclusion and inequality gaps, and strengthening democracy and the common good.

- An ethical commitment to social transformation and construction. These principles are neither exclusive nor contrast with the specificity of University -in terms of a place for the circulation, transfer and construction of knowledge. The idea is to place the political meaning of University in the framework of the social function. In contrast with the conceptions that hold the neutrality of knowledge and science, USC engages and involves HEIs: it allows them to be part of social, political, economic and cultural processes. Being part of it should not be interpreted as being the only one responsible for such processes, but it means that University cannot be thought apart from them.

Moreover, USC involves challenges placed within university processes, so the political meaning of education is in question here. The idea is to identify whether the goal is to provide a collection of knowledge related to certain technical tools which allow for access to productive and service delivery pro- 
Revista de la Escuela de Ciencias de la Educación, año 12, nRo. 11, vol. 2, JUlio a diciembre de 2016. Páginas 177-194. ISSN 1851-6297. ISSN 2362-3349 (EN LiNEA). THe MEANINGS OF UNIVERSITY SOCIAL COMMITMENT. CARINA ANDREA GeRLeRO

cesses, or if it is to develop culture and favor the acquirement of social and cultural goods, or just a combination of both of them.

An attempt to reconstruct the meanings of USC hints at different polysemous concepts that, when applied in relation to USC, create dilemmas as regards their meaning since they are also associated with the political sense of University and education. Furthermore, different attributions of meaning can hinder an identification of the ideological positions which support and give identity to certain concepts, such as pertinence, management, University autonomy, academic freedom and the mission of University, among others.

On this line, the dilemmas now concern the mission of University and the purpose of University management: is the goal to have a work methodology which determines political and economic aims and defines what steps to take so as to achieve that purpose? Or is management thought of as an end in itself concerning control, verification and accountability? In any case, autonomy and academic freedom have both stern supporters and opponents on each end. There are those who see in these concepts the possibility of keeping University away from any external influence, but also those who think that they make the link with society possible.

Ultimately, each proposition externalizes underlying ideologies and political conceptions, whether in their macro or micro-context of development. The strongest challenge is probably to leave behind the surface so as to reach what lies in the depths of each concept, i.e., being able to transcend a clash of ideas based on what "we assume" the other person supports and, in turn, identifying the actual ideology present in the configuration of a particular discourse, regardless of the terms used.

Hannah Arendt (2005, p. 97) expresses that: "If, however, we understand politics to mean a global dominion in which people appear primarily as active agents who lend human affairs a permanence they otherwise do not have, then this hope is not the least bit utopian". Universities and university agents have much to offer by means of contributions which enable taking root in the ideological and political spheres. From the perspective of USC, thought as an expression of the social function of University, there are emerging lines of action intended to democratize access, curricula, research and the transference of knowledge. Furthermore, through USC we can strengthen and expand the concept of extension, especially in terms of the position held on non-University agents; of working on what makes us citizens, on what, at present, determines inclusion and exclusion, and on how collective values operate in a society based on individual competence and economic values.

Another interesting factor worth stressing is HEls' practices from the perspective of SC. This paper does no examine such dimension in detail, since the analysis focuses on the theoretical conceptions and principles behind USC. Nevertheless, there is plenty of bibliographic material available which focuses 
Revista de la Escuela de Ciencias de la Educación, año 12, nRo. 11, vol. 2, Julio a diciembre de 2016. Páginas $177-194$. ISSN 1851-6297. ISSN 2362-3349 (EN LinEA). THE MEANINGS OF UNIVERSITY SOCIAL CommitMENT. CARINA ANDREA GERLERo

on the analysis of practices based on principles related to SC and carried out in different academic contexts. This fact strengthens the idea that we are working on the definition of a field still under construction and that there is much to do and conceptualize about. On the other hand, this is a field constructed in the academic sphere, but also through management and agents' practices. In this regard, it differs from other fields in that it is much more influenced by practices, institutional decisions and geopolitical aspects. As this kind of analysis moves forward, it will certainty contribute to a greater specification of the field and the meaning of USC. Additionally, it may give visibility to how the calling for action made during the CRES and the WCHE was channeled, to the concrete lines of action implemented by HEls and to what extent institutional public policies were transformed as regards teaching, research and extension.

It would appear that the present challenges of HE, in view of the cultural consequences of globalization and changes in social structures, lead to a place where there is nothing else to say or do. Nonetheless, since the $1918 \mathrm{Re}-$ form model was inscribed in Latin American universities, it has called us, even today, to be part of a progressive change of State and society which, based on the defense of democratic life and peace, enables the reconstruction of solidarity-based ties and generates a new appreciation of the Latin American identity and cooperation between the countries of our region.

Assume USC as related to the social function of universities contributes to granting $\mathrm{HE}$ social legitimacy while enabling the organization of university social processes so that actions are sustained by that which is specific of a discipline and by the relation of such discipline with the professional, cultural and social context. In this way, University keeps its essence and, based on its autonomy, it builds exchange processes with society.

\section{Bibliographical Notes}

(1) This article partially communicates the Final Work carried out to obtain the degree of Specialist in High Education Policies and Management awarded by Rosario National University, approved on May 28, 2014. Additionally, it contributes to the definite formulation and development of the Doctoral Thesis Plan: "La formación de profesionales desde la perspectiva del compromiso social universitario" (The education of professionals from the perspective of university social commitment), to be carried out within the PhD in Education of Experimental Sciences, College of Biochemistry and Biological Sciences, Litoral National University (UNL). The fieldwork for the Doctoral Thesis will be carried out within the Project "La formación de profesionales desde la perspectiva del compromiso social. Huellas y olvidos", (The education of professionals from the perspective of university social commitment. Marks and omissions) approved by UNL in the COURSE OF ACTION FOR RESEARCH AND DEVELOPMENT, "R\&D Program Oriented to Social and 
Revista de la Escuela de Ciencias de la Educación, año 12, nRo. 11, vol. 2, Julio a diciembre de 2016. Páginas 177-194. ISSN 1851-6297. ISSN 2362-3349 (EN LinEA). THE MEANINGS OF UNIVERSITY SOCIAL CommitMENT. CARINA ANDREA GERLERO

Productive Problems". 2012 Call.

(2) As the view of Miguel Rojas Mix was taken into consideration during the analysis of the contributions made at the Conference of Rectors, this section does not analyze it in detail.

(3) This Research Project concerns "University Social Commitment" and develops under the scope of the Institute for Studies and Training of the Argentinian National Federation of University Teachers (CONADU), which has an Area of Research whose subject of study is the University.

(4) Additionally, the authors place the origins of the social role of University in the 1918 University Reform.

(5) The authors mention the impacts expected from the application of USR according to Vallaeys' words: Socially responsible management of the organization and its resources; of academic and pedagogic education; of the production of knowledge and promoted epistemological models; and of the participation in SHD. Torres Pernalete \& Trápaga Ortega (2010:79).

\section{Bibliographic References}

- Arendt, H. (2005). The Promise of Politics, New York: Schocken Books. TN: in the original Spanish article, quoted from: Arendt, H. (1997:50). ¿Qué es política?, Barcelona: Paidós.

- Cecchi, N., Lakonich, J., Pérez, D. \& Rotstein, A. (2009). El Compromiso Social de la Universidad Latinoamericana del Siglo XXI: Entre el debate y la acción, IEC-CONADU y ADUM. From: http://www.iecconadu.org.ar/images/LIBRO\%203\%20 El\%20 compromiso\%20Social\%20FINAL.pdf

- Conferencia Mundial de Educación Superior (1998): Comunicado Final. From: http://unesdoc.unesco.org/images/0011/001163/116345s.pdf

- Conferencia Regional sobre Políticas y Estrategias para la Educación Superior en América Latina y el Caribe (1996). Proclamación final. From: http://www.rau. edu.uy/docs/havdecs.htm

- Congreso Internacional de Rectores Latinoamericanos y Caribeños (2007). Declaración final. From: http://www.unesco.org.ve/dmdocuments/3encuentro/ DeclaraCongRectores_Esp.pdf.

Declaración final. From: http://www.cres2008.org/es/index.php.

- De la Cruz Ayuso, C. \& Sasia Santos, P. (2008). La responsabilidad social de la universidad en el proyecto de construcción de una sociedad. Revista Educación Superior y Sociedad. El movimiento de responsabilidad social de la universidad: una comprensión novedosa de la misión universitaria, 13(2). From: http://ess.iesalc.unesco.org. ve/index.php/ess/issue/view/5

- De Sousa Santos, B. (2005). La Universidad en el Siglo XXI. Para una reforma democrática y emancipadora de la Universidad, México: Centro de Investigaciones Interdisciplinarias en Ciencias y Humanidades, Universidad Nacional Autónoma de México. 
Revista de la Escuela de Ciencias de la Educación, año 12, nRo. 11, vol. 2, julio a diciembre de 2016. PÁginas $177-194$. ISSN 1851-6297. ISSN 2362-3349 (EN LinEA). THE MEANINGS OF UNIVERSITY SOCIAL CommitMENT. CARINA ANDREA GERLERo

- Garcia Guadilla, C. (2008). El compromiso social de las universidades [lectronic version]. Cuadernos del Cendes, 25(67), 129-134.

- Margetic, A. \& Suarez, V. (2006). La función social de la Universidad. Consideraciones acerca de los Estatutos de las Universidades Nacionales. Remedios de Escalada: UNLa.

- Regional Conference on Higher Education in Latin America and The Caribbean. (2008). Declaration. From: http://www.iau-hesd.net/sites/default/files/documents/2008_-_declaration_of_the_regional_conference_on_higher_education_in_ latin_americe_and_the_carabean_fr.pdf. TN: in the original Spanish article, quotes from: Conferencia Regional de Educación Superior en América Latina y el Caribe $(2008: 1,6,7)$.

- Rojas Mix, M. (2007). El Compromiso Social de las Universidades de América Latina y el Caribe. From: http://ess.iesalc.unesco.org.ve/index.php/ess/article/ view/46/33

- Saravia, F. \& Escobar, J. (2010). Hacia una democracia socialmente responsable. Una reflexión desde la Universidad Pública. 1st edition, Buenos Aires: Prometeo.

- Torres Pernalete, M. \& Trápaga Ortega, M. (2010), Responsabilidad Social de la Universidad. Retos y Desafíos. 1st edition, Buenos Aires: Paidós.

- Vallaeys, F.; de la Cruz, C. \& Sasia, P. (s, f), Manual de primeros pasos en responsabilización social universitaria. Construyendo ciudadanía en universidades responsables. From: http://www.cyta.com.ar/biblioteca/bddoc/bdlibros/rse/334_ as_ma-nual_rsu_bid.pdf.

- World Conference on Higher Education (2009): The New Dynamics of Higher Education and Research for Societal Change and Development. Comunniqué. From: http://www.unesco.org/fileadmin/MULTIMEDIA/HQ/ED/ED/pdf/WCHE_2009/ FINAL\%20COMMUNIQUE\%20WCHE\%202009.pdf. TN: in the original Spanish article, quote from: Conferencia Mundial de Educación Superior (2009:2-7): Las nuevas dinámicas de la educación superior y de la investigación para el cambio social y el desarrollo. Comunicado Final. From: http://www.unesco.org/education/ WCHE2009/comunicado_es.pdf. 